OPEN ACCESS

\title{
Studying the Interaction of Mass Transport and Electrochemical Reaction Kinetics by Species Frequency Response Analysis
}

To cite this article: Fabian Kubannek and Ulrike Krewer 2020 J. Electrochem. Soc. 167144510

View the article online for updates and enhancements.

\section{Discover the EL-CELL potentiostats}

- Fully independent test channels with Pstat / GStat / EIS

- Optionally with integrated temperature controlled cell chamber

- Unique Connection Matrix: Switch between full-cell and half-cell control at runtime

www.el-cell.com +49 (0) 4079012734 sales@el-cell.com

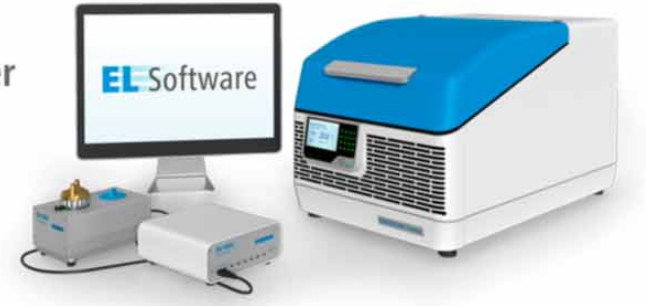




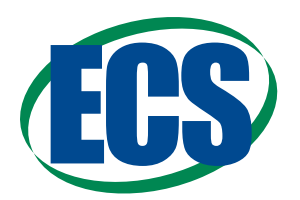

\title{
Studying the Interaction of Mass Transport and Electrochemical Reaction Kinetics by Species Frequency Response Analysis
}

\author{
Fabian Kubannek ${ }^{1}$ (i) and Ulrike Krewer ${ }^{1,2, z_{1}}$ \\ ${ }^{1}$ Institute of Energy and Process Systems Engineering, Technische Universität Braunschweig, Braunschweig, Germany \\ ${ }^{2}$ Institute for Applied Materials, Karlsruhe Institute of Technology, Karlsruhe, Germany
}

Electrochemical macrokinetics contains the interaction of electrode reactions with transport phenomena. To disentangle the individual processes, dynamic techniques such as electrochemical impedance spectroscopy are widely used. Additional information can be obtained when further quantities besides current and potential are recorded. Here, we present and analyze a method to observe the dynamics of the flux of volatile species, i.e. mass transfer, in porous electrodes during electrochemical reactions with a high time resolution. We call this technique species frequency response analysis (sFRA). It is experimentally demonstrated with electrochemical methanol oxidation reaction on a porous $\mathrm{Pt} / \mathrm{Ru}$ electrode. The dynamic relationship between current, potential and the flux of the gaseous reaction product $\mathrm{CO}_{2}$ is measured by differential electrochemical mass spectrometry. The resulting transfer function that relates current density with $\mathrm{CO}_{2}$ flux is analysed in detail by means of a one-dimensional mathematical model. It is demonstrated how the influence of reaction and transport phenomena can be separated in the sFRA Nyquist plot. Practical aspects such as sensitivity and accessible frequency range are discussed as well as the overall prospects and limitations of the technique.

(C) 2020 The Author(s). Published on behalf of The Electrochemical Society by IOP Publishing Limited. This is an open access article distributed under the terms of the Creative Commons Attribution 4.0 License (CC BY, http://creativecommons.org/licenses/ by/4.0/), which permits unrestricted reuse of the work in any medium, provided the original work is properly cited. [DOI: 10.1149/ 1945-7111/abc76e]

Manuscript submitted May 13, 2020; revised manuscript received October 15, 2020. Published November 17, 2020.

Electrochemical reactions at technical electrodes are often influenced by numerous coupled processes. The interactions of reactions, mass transfer and double layer charging render the quantitative description of these systems a complex and challenging task. ${ }^{1}$ A good understanding of all these phenomena is essential for developing better electrodes and electrochemical processes.

Electrochemical impedance spectroscopy (EIS) is a powerful technique that allows one to separate and investigate phenomena on a wide range of time scales. However, processes with similar time constants cannot be distinguished in EIS spectra.

To disentangle such processes, different dynamic techniques have been suggested. One approach that relies on measurements of current and potential, is the use of higher excitation amplitudes which enable the evaluation of the system's response by non-linear frequency response analysis to obtain information on the non-linear system behavior. ${ }^{2,3}$ This technique has been applied for studying fuel cells ${ }^{4-6}$ and recently also for lithium ion batteries. ${ }^{7}$

Recording additional measurement signals is an attractive alternative to employing increasingly complex tools to extract more information from current and potential measurements. In thermoelectrochemical impedance measurements, ${ }^{8,9}$ the electrode temperature is used as an input signal. From the response of current or potential activation energies of electrode reactions and diffusion processes are determined. Photoelectrochemical processes have been studied by intensity-modulated photocurrent spectroscopy, ${ }^{10}$ where a light beam is modulated sinusoidally to separate the contributions to the total photocurrent.

A number of techniques to analyze the relationship of sinusoidal signals of concentrations and electrical quantities have been described in literature. Bessler and colleagues ${ }^{11,12}$ observed the pressure response of a closed metal-air battery that contained gaseous oxygen to analyze the transport of the reactant in the cell and established the term electrochemical pressure impedance spectroscopy. Engebretsen et al. ${ }^{13}$ used the same term for a slightly different approach. They set the pressure inside a Polymer Electrolyte Membrane Fuel Cell (PEMFC) as an input signal and monitored the electrical response. Kulikovsky developed an analytical approach to calculate such spectra by means of linearisation of a non-linear model. ${ }^{14}$ Another approach used for the investigation of

${ }^{\mathrm{z}}$ E-mail: ulrike.krewer@kit.edu a PEMFC is concentration-alternating frequency response analysis (cFRA). ${ }^{15}$ There, the response of current and voltage to perturbations of the oxygen partial pressure were analysed to investigate mass transfer and cathode humidification.

In contrast to these approaches on the cell level, we propose to combine frequency response analysis (FRA) of species flux, i.e. mass transfer, and electrochemical impedance spectroscopy as a tool to separate the influences of different processes on an electrode level. This will yield new insights especially for processes in porous electrodes where the interaction of mass transfer and reaction kinetics, i.e. macrokinetics, can play an essential role. ${ }^{16}$ Because of low response times and the possibility of quantification, Differential Electrochemical Mass Spectrometry (DEMS) is a suitable tool for measuring species fluxes for the purpose of FRA.

In a previous work we have shown how to obtain kinetic data for CO-oxidation in porous electrodes from DEMS experiments using potential step experiments and physical modelling. ${ }^{17}$ DEMS measurements under dynamic conditions include MSCVs, ${ }^{18-20}$ i.e. voltage ramps, step changes in current density ${ }^{17,21}$ and step changes in reactant inlet concentration. ${ }^{22}$ No sinusoidal input signals have been used so far for DEMS, even thought they allow one to cover a broad frequency range and separate processes more accurately.

In this work, DEMS and EIS are combined for the first time. Using the example of the methanol oxidation reaction (MOR), it is demonstrated how to analyze mass transfer and the reaction processes by the combination of electrical and species FRA (sFRA). Please note that we purposefully chose to name it frequency response analysis, not impedance, as impedance implies a complex electric resistance.

First, the new concept of sFRA is explained and approaches for data analysis are developed. Next, the mechanism and kinetics of the MOR as an example reaction is discussed, and the experimental set up for the DEMS measurements is described. A one-dimensional physical model of the processes inside the cell is established which is used for the interpretation of the measurements. Based on the measurements and simulations, transport and kinetic parameters of the porous electrode are identified. Factors influencing sFRA such as amplitude, frequency range and influence of the membrane transport into the vacuum are discussed. A sensitivity analysis shows which parameters can be identified particularly well from the sFRA data. The paper closes with a brief conclusion which focuses on the advantages and limitations of the new technique and outlines further fields of application for sFRA. 


\section{Theory of Species Frequency Response Analysis}

In EIS, the complex impedance value $Z(\omega)=\frac{\Delta U}{\Delta I}$ relates the sinusoidal responses of voltage with amplitude $\Delta U$ to changes in a sinusoidally deflected current with amplitude $\Delta I$. It contains direct information about all processes that affect electrical current and potential such as reaction kinetics, capacitive effects and ohmic resistance. Mass transfer only has an indirect effect on the impedance via changes in concentration which affect the reaction kinetics.

To investigate the interactions of mass transport and electrochemical reaction kinetics by sFRA, we relate the sinusoidal response of the flux, i.e. mass transport, of a species at a certain location close to the electrode $\Delta \dot{n}_{\text {actual }}$ to the sinusoidal change in the electric charge transport $\Delta \dot{n}_{I} . \Delta \dot{n}_{\mathrm{I}}$ is calculated by Faraday's law from the sinusoidal change in current density $\Delta i$ expressed as the theoretical area-specific production rate of that species $\left(\Delta \dot{n}_{\mathrm{I}}=\Delta i z^{-1} \mathrm{~F}^{-1}\right)$. The sFRA transfer function $G_{\text {sFRA }}(\omega)$ mathematically describes the ratio of the amount of species detected after mass transport to the location where $\Delta \dot{n}_{\text {actual }}$ is measured and the species production rate:

$$
G_{\mathrm{SFRA}}(\omega)=\frac{\Delta \dot{n}_{\text {actual }}}{\Delta \dot{n}_{\mathrm{I}}}=\frac{\Delta \dot{n}_{\text {actual }}}{\Delta i z^{-1} \mathrm{~F}^{-1}}
$$

Here, $\mathrm{F}$ is the Faraday constant and $z$ is the number of electrons released or consumed per molecule of product. Even though Faraday's linear relationship between current and reaction rate is one of the most fundamental concepts for steady-state in electrochemisty, the current density actually does not trigger an immediate response of the reaction rate during dynamic operation. Due to the double layer capacitance, adsorption processes or side reactions, the dynamic response of the actual production rate of a species $\alpha$ will be shifted. When the flux of the product species is measured closely to a porous electrode, the transport inside the porous electrode and between the electrode and the point of measurement will induce another shift in the response signal. This means that information on reaction kinetics and electrode transport properties is contained in the sFRA spectrum. Figure 1 schematically shows this concept.

Due to the normalization of the current signal, the transfer function $G_{\mathrm{sFRA}}$ is dimensionless, independent of electrode area and of number of transferred electrons per molecule of the measured compound. $G_{\mathrm{sFRA}}$ approaches a maximum value of one for the frequency approaching a value of zero, if no side reactions occur and if all product molecules arrive at the location where $\Delta n_{\text {actual }}$ is determined. If the magnitude of $G_{\mathrm{SFRA}}$ is very small, a large part of the current is used for double layer charging, production of intermediates or side reactions in the respective frequency range. If $G_{\text {sFRA }}$ is larger than one, dynamic processes amplify the change in production rate so that $\Delta \dot{n}_{\text {actual }} \geqslant \Delta i z^{-1} \mathrm{~F}^{-1}$. Further characteristic properties of the transfer function will be discussed in the next section specifically for the MOR.

The sFRA transfer function can be experimentally determined using DEMS, which allows one to measure fluxes of volatile substances with a sufficient time resolution. The measured ion current of the mass spectrometer is directly proportional to the amount of substance entering the vacuum system over a wide ion current range so that quantification is straightforward. ${ }^{17}$ The transfer function $G_{\mathrm{MS}}(\omega)$ is obtained from experimental data by dividing the sinusoidal signals of ion current $I_{\mathrm{MS}}$ and current $I . \Delta I_{\mathrm{MS}}$ is the signal of the sinusoidal ion current measured by the mass spectrometer at a given mass to charge ratio in the frequency domain over the frequency $\omega$.

$$
G_{\mathrm{MS}}(\omega)=\frac{\Delta I_{\mathrm{MS}}(\omega)}{\Delta I(\omega)}
$$

The same mathematical transformation procedures that are used to calculate EIS spectra by converting current and voltage signals from the time domain into the frequency domain such as Fast Fourier Transform can be used.

$G_{\mathrm{MS}}(\omega)$ can be readily transformed into the sFRA transfer function: The MS signal is converted into the molar flux that enters the vacuum $\dot{n}_{\mathrm{MS}}$ using the electrode area $A$ and the MS calibration constant $K^{*}$ that is determined as explained in Ref. 17. The following equation relates $G_{\mathrm{MS}}(\omega)$ and $G_{\mathrm{SFRA}}(\omega)$ :

$$
G_{\mathrm{SFRA}}(\omega)=\frac{\Delta \dot{n}_{\mathrm{MS}}}{\Delta \dot{n}_{\mathrm{I}}}=\frac{\Delta I_{\mathrm{MS}}}{K^{*} A} \frac{z \mathrm{~F} A}{\Delta I}=G_{\mathrm{MS}}(\omega) \frac{z \mathrm{~F}}{K^{*}}
$$

For measurements with DEMS and assuming no side products, the magnitude of $G_{\mathrm{sFRA}}$ at a limiting frequency of $0 \mathrm{~Hz}$ shows the share of product that enters the vacuum system and equals the DEMS collection efficiency $N .^{17}$

Here, the anodic current direction is defined as positive, and the compound of interest is a product of an electrochemical oxidation reaction, which $\mathrm{CO}_{2}$ is in case of the MOR. If a reactant is measured, the production rates become consumption rates and the sign of the calculated transfer function changes when using the same equations. This is also the case if an electrochemical reduction takes place because the sign of the current changes.

Similar as for EIS, the current as sinusoidal input signal may be replaced with a sinusoidal potential signal. This however results in a different transfer function. The representation from Eq. 1 seems more practical though for the purpose of separating reaction and transport phenomena because reaction rates and mass transfer are directly related to the current and only indirectly related to the potential.

The frequency range and the expected signal magnitudes can be estimated from the geometry of the measurement cell and the transport parameters. In DEMS, a PTFE membrane separates the vacuum system from the electrolyte. It is typically $50 \mu \mathrm{m}$ thick. When the electrode is placed directly at the membrane and transport within the electrode is neglected, the time constant of the membrane transport can be roughly estimated by $\tau=L^{2} / D$. For $\mathrm{CO}_{2}$ $\left(D=1.92 \mathrm{~m}^{2} \mathrm{~s}^{-1}\right) \tau=1.88$ results. The magnitude of the signal, i.e. the normalized amount of substance entering the vacuum system, depends on the DEMS collection efficiency $N$. Typically, values between 0.5 and 0.9 are reached in a DEMS setup where the porous electrode is in direct contact with the membrane. Accordingly, the sinusoidal signal of the molar flux measured by the MS is expected to exhibit a magnitude between $50 \%$ and $90 \%$ of the reaction rate's amplitude and a minimum phase shift corresponding to a time constant of approximately two seconds.

\section{Methanol Oxidation Reaction}

In this section we explain why the MOR was chosen for demonstration of the new frequency response analysis technique, and the reaction mechanism will be discussed. MOR kinetics on various catalysts have already been analysed intensely in literature for model electrodes ${ }^{23-25}$ as well as technical electrodes. ${ }^{26-28}$ In the latter three references, mathematical models have been developed that describe current-potential relationships for a carbon supported $\mathrm{Pt} / \mathrm{Ru}$ catalyst under steady state and dynamic conditions. This type of catalyst is also employed here because of its relevance for application in fuel cells. The electrochemical oxidation reaction can be described by the following steps ${ }^{26,29}$ :

$$
\begin{gathered}
\mathrm{CH}_{3} \mathrm{OH}+\mathrm{Pt} \cdot \stackrel{r_{1}}{\rightarrow} \mathrm{CO}_{\mathrm{ad}}+4 \mathrm{H}^{+}+4 \mathrm{e}^{-} \\
\mathrm{H}_{2} \mathrm{O}+\mathrm{Ru} \cdot \stackrel{r_{2}}{\leftrightarrow} \mathrm{OH}_{\mathrm{ad}}+\mathrm{H}^{+}+\mathrm{e}^{-} \\
\mathrm{CO}_{\mathrm{ad}}+\mathrm{OH}_{\mathrm{ad}} \stackrel{r_{3}}{\rightarrow} \mathrm{CO}_{2}+\mathrm{H}^{+}+\mathrm{e}^{-}+\mathrm{Pt} \cdot+\mathrm{Ru} \cdot
\end{gathered}
$$

The stable adsorbate on the Pt surface is $\mathrm{CO}$ which further reacts with $\mathrm{OH}$ adsorbed on $\mathrm{Ru}$ to $\mathrm{CO}_{2}$. It is assumed that water only 


\section{species Frequency Response Analysis: $\quad G_{S F R A}=\frac{\Delta \dot{n}_{\alpha}}{\Delta \dot{n}_{\mathrm{i}}}$}

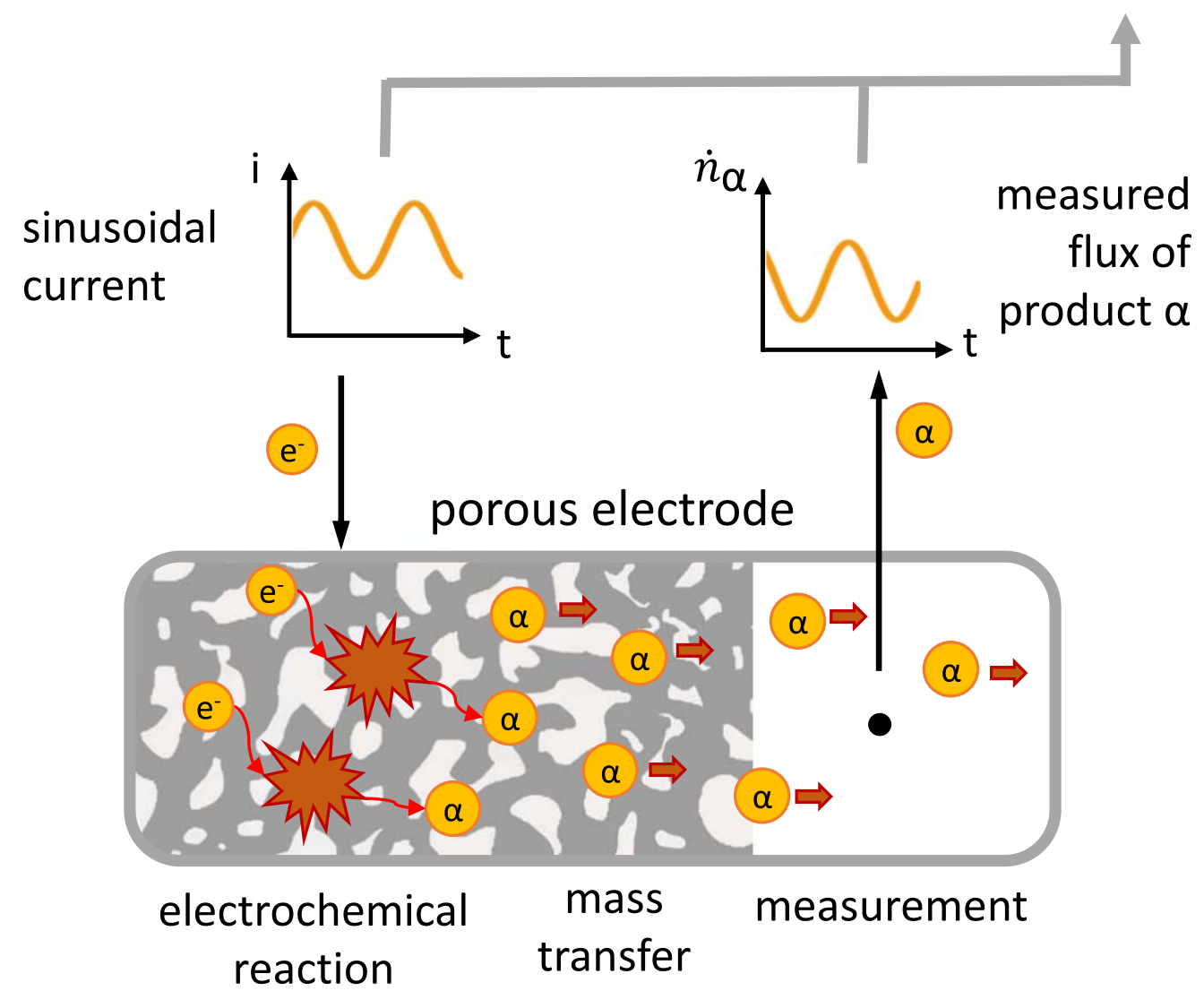

Figure 1. Schematic diagram showing how sinusoidal current and species flux signals are combined in sFRA spectra. The spectra contain information on kinetics and mass transfer properties such as reaction rate constants, electrode porosity and effective diffusion coefficients.

chemisorbs on free $\mathrm{Ru}$ sites ( $\mathrm{Ru} \cdot$ ) and methanol partially oxidises to $\mathrm{CO}$ only on free Pt sites (Pt.).

The focus and novelty of the work presented in this work lies in the methodology rather than in gaining new insights into the MOR reaction mechanism. In technical electrodes, $\mathrm{CO}_{2}$ is the only product of the MOR. It can be detected well by DEMS and its transport properties inside the electrode are highly important for direct methanol fuel cells because they determine the transition between single-phase and two-phase flow on the anode side. Furthermore, literature models that help one to analyze and interpret experimental data are available. All these points render the MOR a suitable example system for the application of sFRA.

\section{Experimental}

Porous electrodes were produced by spray coating $5 \mathrm{~g} \mathrm{~m}^{-2}$ of a 1:1 Pt/Ru catalyst (Johnson Matthey, HiSpec 10000) with Nafion solution $(5 \% \mathrm{w} / \mathrm{w}$ of catalyst weight, Qintech NS05) directly on a porous PTFE membrane $(0.2 \mu \mathrm{m}$ pore size, Pall $)$, resulting in a layer thickness of $25 \mu \mathrm{m}$ determined by SEM measurements. They were mounted in a cyclone-flow cell specifically designed for DEMS experiments under defined mass transfer conditions. $\mathrm{CO}_{2}$ produced at the electrode diffuses through the PTFE membrane into a vacuum system, which is connected to the bottom of the cell. In the vacuum system, $\mathrm{CO}_{2}$ is detected by a mass spectrometer. The PTFE membrane prevents the breakthrough of liquid electrolyte into the vacuum system.

The cyclone-flow DEMS cell which was used to record MSCV and sFRA spectra is depicted in Fig. A.1. The cell and the instrumental set up have been described previously. ${ }^{17}$ Electrode production, the $\mathrm{Pt} / \mathrm{Ru}$ catalyst and the fluid flow inside the cell as well as the collection efficiency are discussed in detail there.

The electrolyte that was circulated through the cyclone-flow DEMS cell at a constant flow rate of $230 \mathrm{ml} \mathrm{min}^{-1}$ contained $0.5 \mathrm{~mol}^{-1}$ methanol (VWR, HPLC grade) and $0.25 \mathrm{~mol}^{-1}$ $\mathrm{HClO}_{4}$ (Sigma Aldrich, ACS grade). No further methanol was added during the experiments because from the current density it was calculated that methanol consumption is negligible over the duration of the experiment. All measurements were carried out at a constant room temperature of $298 \mathrm{~K}$.

Two types of dynamic electrochemical experiments were carried out: cyclic voltammetry and electrochemical impedance spectrometry. All potentials are reported with respect to a reversible hydrogen electrode. During all experiments current, voltage and the mass spectrometer's ion current signal at a mass to charge ratio $\mathrm{m} / \mathrm{z}$ of 44 were recorded. At $\mathrm{m} / \mathrm{z}=44$ the expected main product $\mathrm{CO}_{2}$ can be detected. A Reference 3000 potentiostat (Gamry) and a PrismaPlus QMG 220 mass spectrometer (Pfeiffer Vacuum) were used. The potentiostat's inbuilt EIS function was not used because it automatically transforms the current and voltage data into the frequency domain. After that it cannot be correlated to the MS signal any more. Thus the spectra were obtained using using a Labview program that was developed specifically for this purpose. Sinusoidal input current signals of different frequencies were applied to the working electrode. The responses of potential and ion current were recorded in the time domain and transformed to the frequency domain by a fast Fourier transform. 


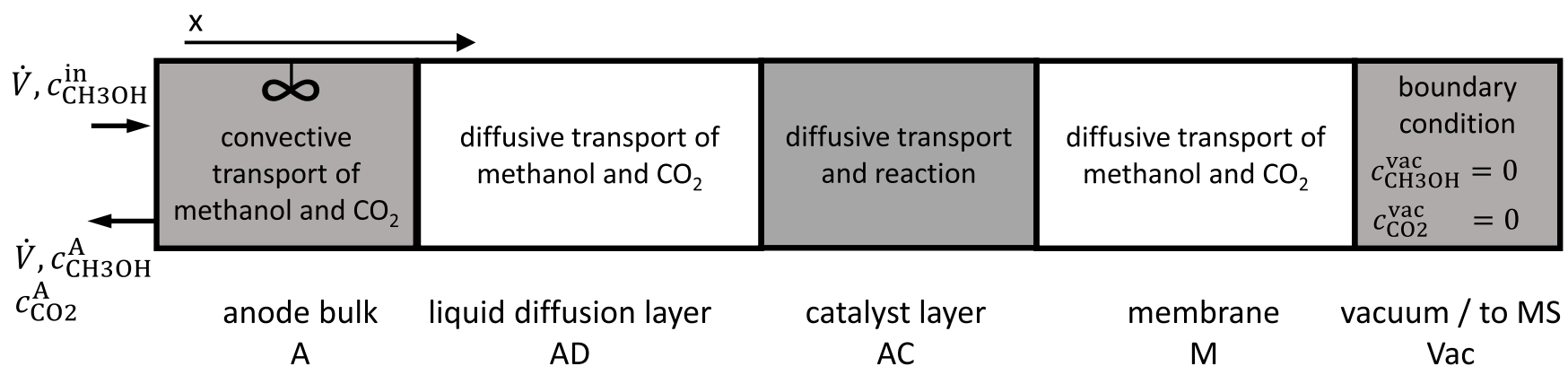

Figure 2. Schematic overview of the structure of the mathematical model used for the evaluation the of sFRA spectra.

EIS and sFRA spectra were recorded in parallel in potentiostatic mode using an amplitude of $50 \mathrm{mV}$ and a constant potential offset of $0.7 \mathrm{~V}$ in the frequency range from $0.02-1 \mathrm{~Hz}$. The reason for choosing this comparatively high excitation amplitude and its effect on the linearity of the system are discussed in the results section. The experimental transfer function was calculated from the response signal of current $\Delta I$ and ion current $\Delta I_{\mathrm{MS}}$ to the potential signal $\Delta U$ by Eq. 4.

$$
G_{\mathrm{MS}}(\omega)=\frac{\Delta I_{\mathrm{MS}}}{\Delta I}=\frac{\Delta I_{\mathrm{MS}}}{\Delta U} \frac{\Delta U}{\Delta I}
$$

Equation 4 requires the system to be linear which is assumed here.

$\mathrm{CV}$ s were recorded between $0.45 \mathrm{~V}$ and $0.8 \mathrm{~V}$. The second cycle is shown. This voltage range is chosen because below $0.45 \mathrm{~V}$ there is only a small amount of $\mathrm{CO}_{2}$ produced, and above $0.8 \mathrm{~V}$ ruthenium dissolves.

\section{Modelling}

A one-dimensional physical model for the processes inside the cell has been developed to lay the basics for understanding sFRA in general and be able to analyze the measurements. The interpretation of classical EIS data is often not straightforward because the impact of processes can overlap in the same frequency range and the time constants of individual processes are unknown. However, various examples from literature have demonstrated the advantages of employing physical models to interpret EIS spectra. ${ }^{5,26,30}$ For the interpretation of species sFRA spectra modelling is essential because no spectra and their interpretations are available in literature.

The structure of the model and the considered processes are depicted in Fig. 2. The bulk volume, i.e. the cyclone cell's electrolte filled volume, is assumed to be well-mixed because of the electrolyte convection. ${ }^{17}$ From the bulk, methanol diffuses through the liquid diffusion layer into the catalyst layer where the reactions take place. Additionally, methanol evaporates through the porous PTFE membrane. In the catalyst layer, balance equations for the surface species describe the change of surface coverage by the reaction intermediates. $\mathrm{CO}_{2}$ produced in the catalyst layer can either diffuse through the membrane into the vacuum system or through the diffusion layer into the bulk. Partial differential equations for the transport in diffusion layer, catalyst layer and membrane are discretised by the finite volume method. Overall, the transport is described similarly as in our previously published work ${ }^{17}$ because the same DEMS cell is used. The description of the reaction kinetics follows a previously published non-linear physical model of a direct methanol fuel cell by Krewer et al. ${ }^{28}$ which is based on the MOR mechanism shown above. The reaction rate constants and the diffusion coefficients of methanol and $\mathrm{CO}_{2}$ in the PTFE membrane as well as the number of active sites in the catalyst layer were identified from experimental data. A detailed model description including equations, model assumptions, the parameter identification procedure using optimization algorithms, the parameter values and the model validation can be found in the Appendix.

\section{Results and Discussion}

In this section the sFRA spectra are analysed in detail and interpreted using insights from the simulation. First, technical aspects are covered. It will be examined if the comparatively small experimental frequency range from 0.02 to $1 \mathrm{~Hz}$ is suitable for investigating the MOR macrokinetics. Also, the influence of the excitation amplitude will be analysed. The influence of the sensitivity and background noise of the mass spectrometer on the frequency range that can be measured will be evaluated systematically.

Next, the contributions of reaction and transport processes of the MOR on Pt/Ru in the DEMS cell will be analysed using the FRA spectrum. The origin of the negative real and imaginary part of the spectrum will be explained and assigned to specific processes. Finally, a sensitivity analysis will show which parameters influence the spectra and may thus be experimentally accessible through the technique.

Electrochemical impedance and species frequency response analysis spectra.--In Fig. 3, the MS signal measured at 20, 80 and $200 \mathrm{mHz}$ is shown. With increasing frequency the signal amplitude decreases and the phase shift between the current and the ion current increases. The transport from the electrode to the vacuum system apparently causes a delay between current and mass spectrometer signal. Additionally it has a dampening effect on the amplitude at higher frequencies. In Fig. 4, the experimental and the simulated EIS and sFRA Nyquist plots are compared. Only the first harmonic, which corresponds to the linear part of the response signal, is analysed here. The agreement between experiment and simulation is reasonable for

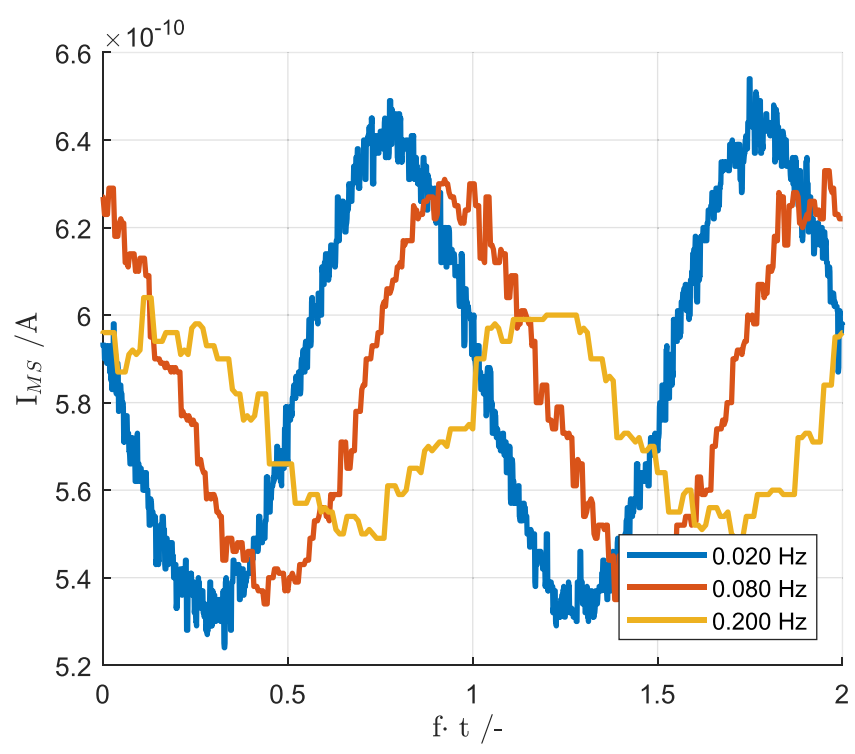

Figure 3. MS signal during cFRA measurements at 20, 80 and $200 \mathrm{mHz}$ The $x$-axis is normalised by the respective frequency. 

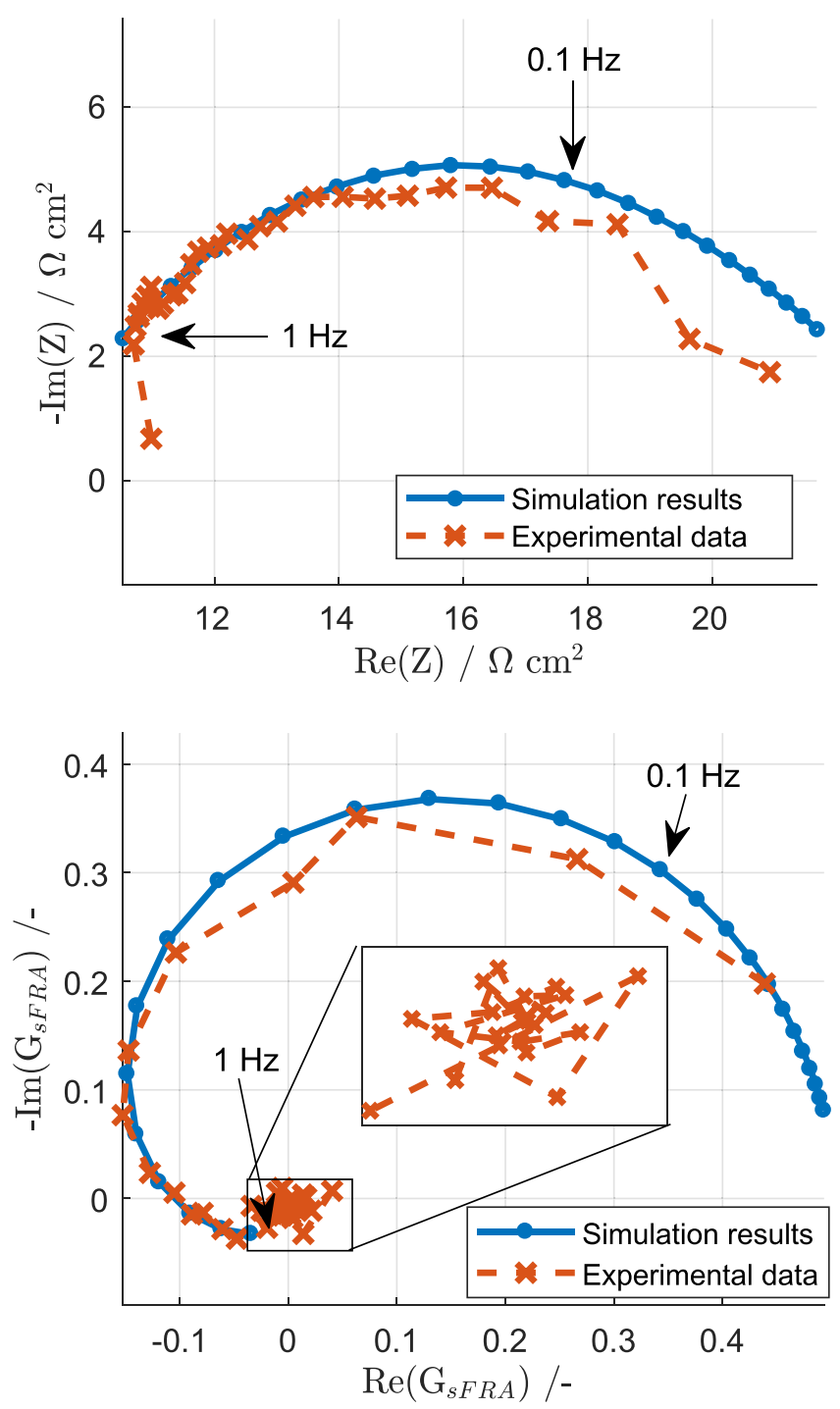

Figure 4. Comparison of experimental and simulated Nyquist plots for EIS (top) and sFRA (bottom). Frequency: $0.02-1 \mathrm{~Hz}, c_{\mathrm{MeOH}}=0.5 \mathrm{~mol}^{-1}$ in $0.1 \mathrm{~mol}^{-1} \mathrm{HClO}_{4}, 0.5 \mathrm{mg} \mathrm{cm}^{-2} \mathrm{Pt} / \mathrm{Ru}$ on carbon, $\mathrm{T}=25^{\circ} \mathrm{C}$.

both EIS and sFRA. The Nyquist plot of the EIS shows one large distorted semicircle that comprises the reactions and double layer charging as well as diffusion at lower frequencies. The semicircle contains both reaction and diffusion phenomena. This can be rationalized when comparing the diffusion time constant for mass transfer in the measurement cell with the reaction time constant reported in literature. The diffusion time constant for methanol diffusion through the concentration boundary layer $\tau$ can be estimated as $\tau=\left(\delta^{\mathrm{DL}}\right)^{2} / D_{\mathrm{MeOH}}=110 \mu \mathrm{m}^{2} / 0.84 \cdot 10^{-9} \mathrm{~m}^{2} \mathrm{~s}^{-1}=14.1 \mathrm{~s}$ and corresponds to a frequency slightly below $0.1 \mathrm{~Hz}$. In the literature, reaction time constants in the same order of magnitude are reported for the MOR. ${ }^{27,28}$ There is no linear increase in impedance at low frequencies (Warburg-Impedance) because the convection in the cell limits the thickness of the concentration boundary layer $\delta^{\mathrm{DL}}$. EIS spectra of the MOR often show an inductive loop at low frequencies, which is caused by surface processes. ${ }^{26}$ This loop is not visible here, probably because of overlaying transport phenomena and, possibly, capacitive currents. As explained above, methanol mass transport has a similar time constant as the reaction processes which would cause the inductive loop. One reason for a large double layer capacitance contribution could be the fact that a part of the GDL-electrode below the gasket might be flooded with electrolyte. This part of the electrode is not active because no methanol is transported there but might contribute to the double layer capacity. Furthermore, it has been shown by Krewer et al. ${ }^{26}$ that the size of the inductive loop depends on the $\mathrm{Pt} / \mathrm{Ru}$ ratio at the surface and is not always present.

The experimental data show some noise at higher frequencies which might be caused by the use of the in-house Labview program that does not contain elaborate noise filtering and is not optimized for data acquisition speed. Specifically, the data acquisition speed of the Labview program was limited by the time for a loop execution during which data points were read, processed, and plotted. The accuracy and speed of the data acquisition is high enough for our purposes though because we focus on transport processes whose dynamics are not visible at high frequencies and because literature shows that the dynamics of the MOR lie within the accessible frequency range. ${ }^{28}$

The experimental Nyquist plot from the sFRA curve shows two characteristic features which are both reproduced by the simulation. First, the imaginary part of the transfer function is positive at high frequencies. Second, the real part of the transfer function is negative at intermediate to high frequencies. The reason for this behavior is discussed below.

In conclusion, sFRA spectra can be recorded by DEMS measurements. Both the phase shift and the amplitude are monotonic functions of the frequency. The phase shift continuously increases with frequency, the amplitude decreases down to zero at an infinite frequency, making the sFRA transfer function a strictly proper transfer function.

Frequency range for species frequency response analysis.-In this section we discuss which frequency range is suitable for investigating the MOR by sFRA. The experimental frequency range from 0.02 to $1 \mathrm{~Hz}$ is very narrow compared to EIS where typically frequencies between $1 \mathrm{mHz}$ and $1 \mathrm{MHz}$ are used. Thus a wider frequency range is simulated for sFRA and EIS using the validated model to investigate if any important features of the curve have been missed out. Figure 5 shows the EIS and sFRA Nyquist plot for an extended frequency range of $0.0003-10 \mathrm{~Hz}$. The experimental frequency range and some frequency points are highlighted for clarity. It can be seen that the sFRA curve does not show any significant features outside the experimental frequency range but quickly converges to zero at high frequency and intercepts with the real axis at low frequency. The EIS Nyquist plot approaches the real axis for higher and lower frequencies. The DEMS measurements are thus fast enough to cover the major effects and time constants of the electrode processes, and the experimentally accessible frequency range is sufficient to investigate the MOR in the current set up. For different set ups or reactants such as hydrogen, which diffuses much faster than $\mathrm{CO}_{2}$, this analysis would need to be confirmed though.

Relationship between noise of MS signal and maximum frequency.-While the simulated sFRA curves are smooth, the experimental MS signal data contains noise. The background noise of the MS is constant. Thus, at higher frequencies, where the magnitude of the transfer function and thus the absolute amplitude of the MS signal is smaller, a constant noise level is relatively more severe. When the noise level exceeds the MS signal's amplitude, the signal does not contain sufficient information to be transformed into the frequency domain correctly and random data points are produced by the fast Fourier transform. This effect can be seen in the inset of Fig. 4 where the experimental data points are masked by strong noise at high frequencies. Thus, the following condition must be met:

$$
\begin{gathered}
\left|\Delta I_{\mathrm{MS}}\right|=\left|G_{\mathrm{MS}}\right||\Delta I| \stackrel{!}{>} P_{\mathrm{N}, \mathrm{MS}} \\
\left|G_{\mathrm{MS}, \mathrm{min}}\right| \stackrel{!}{>} \frac{P_{\mathrm{N}, \mathrm{MS}}}{|\Delta I|}
\end{gathered}
$$

The magnitude of the transfer function $\left|G_{\mathrm{MS}}\right|$ must be larger than the ion current noise $P_{\mathrm{N}, \mathrm{MS}}$ divided by the ion current signal amplitude. 

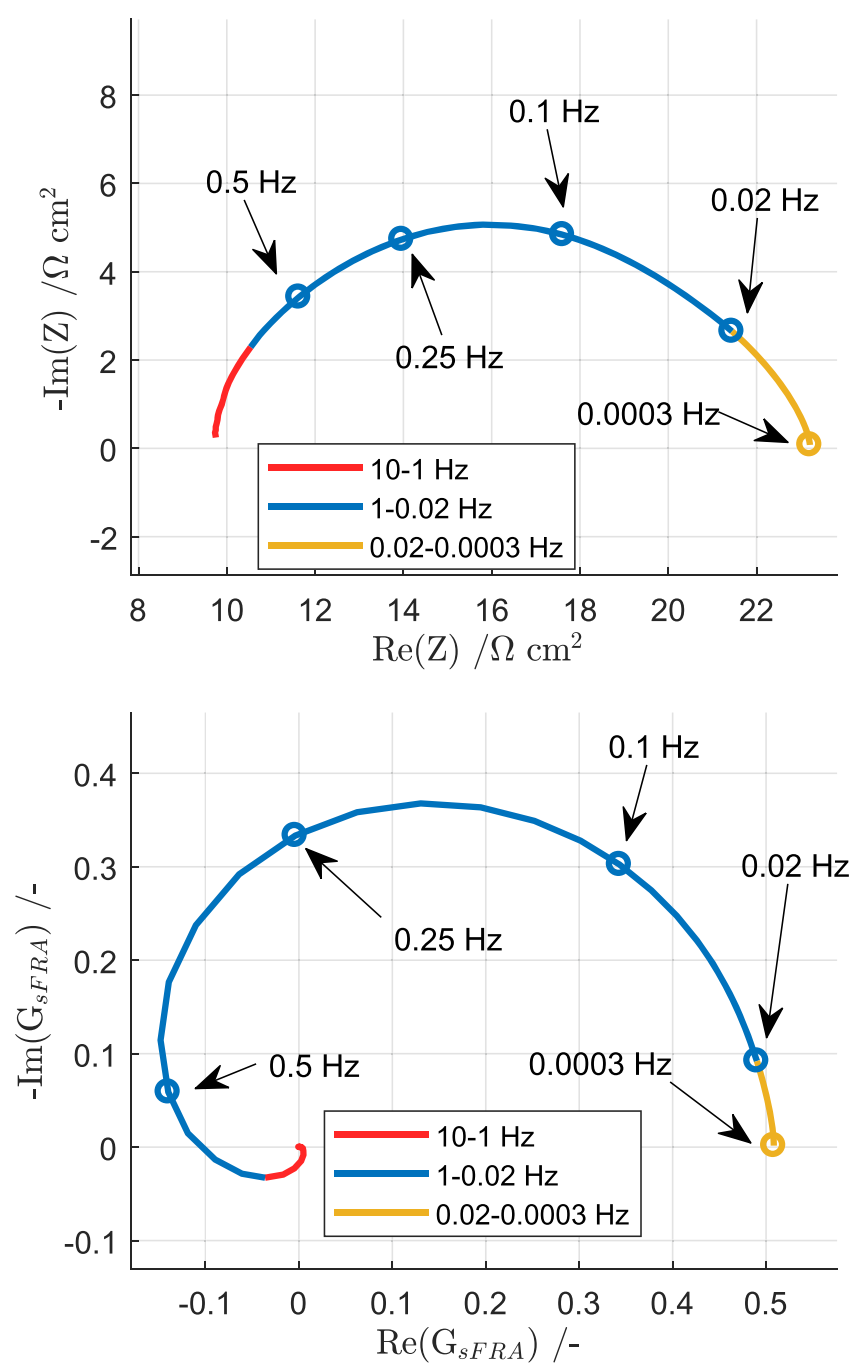

Figure 5. Simulated EIS (top) and sFRA (bottom) Nyquist diagram in an extended frequency range of $0.0003-10 \mathrm{~Hz}$ showing that the characteristic features of the sFRA Nyquist curve are within the experimental frequency range of $0.02-1 \mathrm{~Hz}$.

From the MS signal in the time domain, a noise level of approximately $20 \mathrm{pA}$ is determined. In Fig. $6 \mathrm{a},\left|G_{\mathrm{MS} \text {,min }}\right|$ is depicted in the sFRA Nyquist plot for two different values of $\Delta I$. For all points within the circle, the current $I_{\mathrm{MS}}$ corresponding to the amplitude of the $\mathrm{CO}_{2}$ flux into the vacuum is smaller than the noise of the MS signal. Thus, to record the characteristic features of the spectrum near the origin, high amplitudes are required.

Since the magnitude of the species frequency response response function $\left|G_{\mathrm{MS}, \mathrm{n}}\right|$ is a function of the frequency $\omega$, the maximum frequency can be predicted for given input current amplitudes and MS background noise levels with the simulation model. In Fig. 6b, the maximum attainable frequency is plotted over the amplitude of the current input signal for three different noise levels: $10 \mathrm{pA}, 20 \mathrm{pA}$, and $40 \mathrm{pA}$. The maximum frequency increases with input signal amplitude in all cases. The shape of the curve is convex, meaning that the benefits of higher input signals diminish at higher input signal magnitudes.

The predicted values agree reasonably well with the experimental values. According to the simulations, the maximum frequency for a meaningful spectrum is $0.6 \mathrm{~Hz}$ at a current amplitude of $3 \mathrm{~mA}$. The maximum frequency in the experiment is approximately $0.55 \mathrm{~Hz}$ at a current amplitude of $3.2 \mathrm{~mA}$.

The input signal magnitude in Figs. 7 and 6 are reported in absolute values. This representation was chosen because the signal to noise ratio of the MS depends on the absolute species flow rather than on the area specific values. Furthermore, the electrode area cannot be scaled easily in the DEMS set up since the vacuum pumps have a limited capacity. However, the current amplitude can be normalised by the electrode area of $0.78 \mathrm{~cm}^{2}$ if desired.

Non-linearity at high input current magnitude.-As shown in the previous section, the amplitude of the current may not be reduced below a certain threshold because otherwise the MS signal cannot be discerned from the background noise. In this section, the influence of the current amplitude on the system's linearity is analyzed. Based on the condition for linear systems $f(a \cdot x)=a \cdot f(x)$ we examine if $\Delta \dot{n}_{\mathrm{MS}}(a \Delta I)=a \Delta \dot{n}_{\mathrm{MS}}(\Delta I)$. This is the case when $G_{\mathrm{sFRA}}(\omega)=$ $\frac{\Delta I_{\mathrm{MS}}}{K^{*} \mathrm{z}} \frac{\mathrm{FA}}{\Delta I}$ is independent of the amplitude of the current.

Figure 7 shows the sFRA Nyquist plot simulated with three different current amplitudes: $0.1,2$ and $3 \mathrm{~mA}$. The curves at $0.1 \mathrm{~mA}$ and $2 \mathrm{~mA}$ are almost identical, whereas the curve at $3 \mathrm{~mA}$ show a small deviation at intermediate frequencies. From the deviation between the curves at 0.1 and $3 \mathrm{~mA}$ it is concluded that amplitudes of $3 \mathrm{~mA}$ some non-linear effects are present.

What does this mean for the given experimental conditions? In the experiment, the amplitude of the potential perturbation is controlled by the potentiostat and is constant. The amplitude of the current signal changes with the frequency because the magnitude $|Z|$ of the EIS impedance $Z=\frac{\Delta U}{\Delta I}$ changes with the frequency. At $1 \mathrm{~Hz}$ the amplitude of the current signal is $3.6 \mathrm{~mA}$, and at $0.02 \mathrm{~Hz}$ it is $1.8 \mathrm{~mA}$. In the intermediate frequency range, where non-linear behavior might have an influence, the current amplitude is $\approx 2 \mathrm{~mA}$. The difference between the curves for a current amplitude of 0.1 and $2 \mathrm{~mA}$ (compare Fig. 7) is very small and much lower than the experimental inaccuracy. Therefore, we treat the system as linear.

Separation of transport and reaction processes.-In this section, the contributions of the individual processes to the sFRA spectrum will be discussed. In order to explain the shape of the Nyquist plot and gain a better understanding of the processes that determine the electrode behavior, transfer functions for the sub-processes are analysed. As explained above, the system behavior can be approximated as linear. Thus, the transfer function can be split up into the contributions of relevant processes:

$$
G_{\mathrm{SFRA}}=\frac{\Delta \dot{n}_{\mathrm{MS}}}{\Delta \dot{n}_{\mathrm{I}}}=\underbrace{\frac{\Delta \dot{n}_{\mathrm{R}}}{\Delta \dot{n}_{\mathrm{I}}}}_{G_{\mathrm{I} / \mathrm{R}}} \cdot \underbrace{\frac{\Delta \dot{n}_{\mathrm{M}}^{\text {in }}}{\Delta \dot{n}_{\mathrm{R}}}}_{G_{\mathrm{R} / \mathrm{M}}} \cdot \underbrace{\frac{\Delta \dot{n}_{\mathrm{MS}}}{\Delta \dot{n}_{\mathrm{M}}^{\text {in }}}}_{G_{\mathrm{M} / \mathrm{MS}}}
$$

$G_{\mathrm{M} / \mathrm{MS}}=\frac{\Delta \dot{n}_{\mathrm{MS}}}{\Delta \dot{n}_{\mathrm{M}}}$ is the transfer function relating the flux into the mass spectrometer's vacuum system to transport through the membrane. $\Delta \dot{n}_{\mathrm{MS}}$ is the flux of $\mathrm{CO}_{2}$ into the vacuum and $\Delta \dot{n}_{\mathrm{M}}^{\text {in }}$ is the flux of $\mathrm{CO}_{2}$ that enters the membrane from the catalyst layer. $G_{\mathrm{I} / \mathrm{R}}=\frac{\Delta \dot{n}_{\mathrm{R}}}{\Delta \dot{n}_{\mathrm{I}}}$ is the transfer function relating the $\mathrm{CO}_{2}$ producing reaction to the current. $\Delta \dot{n}_{\mathrm{R}}=\int_{0}^{\delta^{\mathrm{AC}}} \frac{r_{3}}{\delta^{\mathrm{AC}}} d x$ is the rate of $\mathrm{CO}_{2}$ production calculated by integrating the local rate of $\mathrm{CO}_{2}$ production $r_{3}$ over the catalyst layer thickness $\delta^{\mathrm{AC}} . \Delta \dot{n}_{\mathrm{I}}$ is the charge transport expressed as the theoretical rate of $\mathrm{CO}_{2}$ production calculated from the current by Faraday's law. $G_{\mathrm{R} / \mathrm{M}}=\frac{\Delta \dot{n}_{\mathrm{M}}^{\text {in }}}{\Delta \dot{n}_{\mathrm{R}}}$ is the transfer function relating the $\mathrm{CO}_{2}$ production to the transport into the membrane.

Such a decomposition of transfer functions is a common procedure in control engineering, but was also used for EIS $^{26}$ and steps in current density. ${ }^{31}$ However, a physical analysis has seldom been done in electrochemistry.

In Fig. 8a, the reaction transfer function $G_{I / R}$ is depicted. The curve starts at the origin of the coordinate system at high frequencies. The reason for this is the fact that at high frequencies the input current entirely goes into double layer charging and discharging so that no change in the $\mathrm{CO}_{2}$ production is induced and the magnitude of the transfer function is zero. 


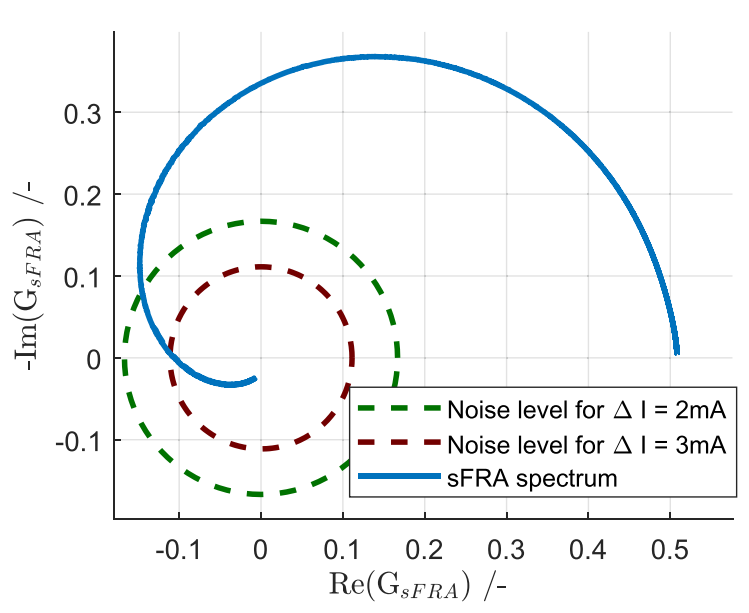

(a)

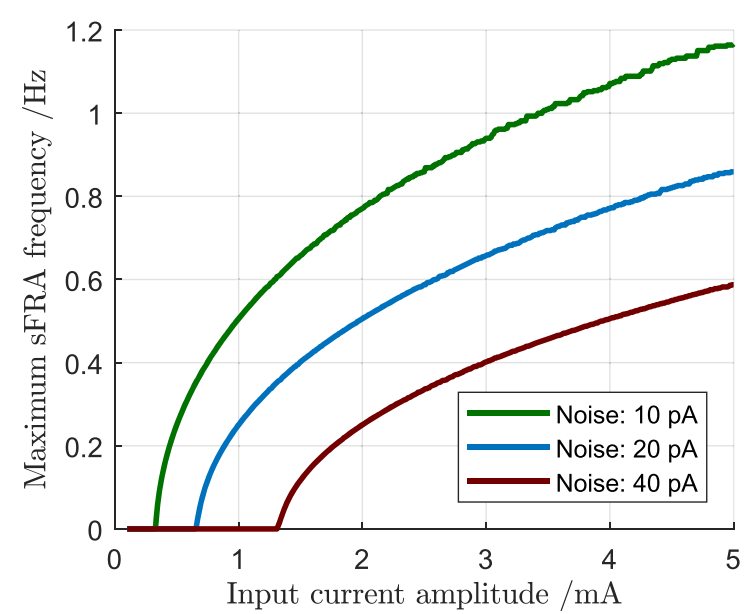

(b)

Figure 6. Left: sFRA Nyquist plot with noise circles for different input current signal amplitudes in a frequency range of $0.002-10 \mathrm{~Hz}$. Right: Calculated maximum frequency for sFRA over input current signal magnitude for different MS signal noise levels. The blue line corresponds to the experimental conditions. The curves are not smooth at high input current amplitudes because the spectra are computed for a finite number of discrete frequencies.

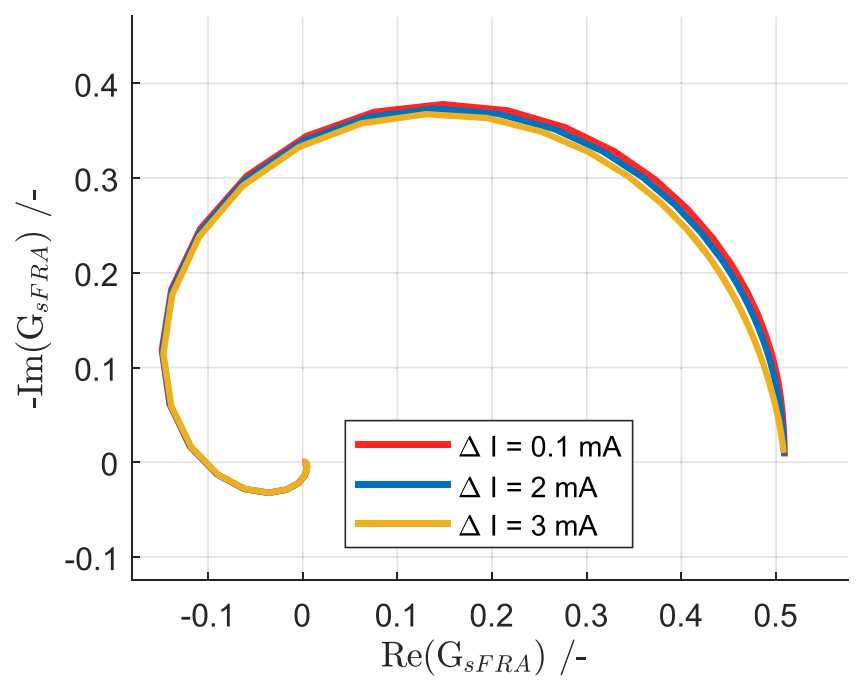

Figure 7. Simulated sFRA Nyquist diagram in an extended frequency range from $0.0003-10 \mathrm{~Hz}$ for input amplitudes between 0.1 and $3 \mathrm{~mA}$ showing that the influence of non-linearities is small.

At low frequencies, the curve approaches the real axis and the magnitude becomes one. In this range, dynamic effects can be neglected and $\mathrm{CO}_{2}$ production equals the value predicted by Faraday's law. This reflects the fact that the model does not account for any side reactions. The magnitude of the transfer function is larger than one in an intermediate frequency range. This means that the $\mathrm{CO}_{2}$ production increases and decreases stronger with the current than predicted by Faraday's law. The reason for this is the interplay of surface processes: During the positive half-wave of the current input signal, the surface coverage of adsorbed $\mathrm{CO}$ decreases, causing a disproportionally large increase in $\mathrm{CO}_{2}$ production because only two electrons are needed to oxidize the adsorbed $\mathrm{CO}$ to $\mathrm{CO}_{2}$. Thereby $G_{\mathrm{I} / \mathrm{R}}=\frac{\Delta \dot{n}_{\mathrm{R}}}{\Delta \dot{n}_{\mathrm{I}}}$ exceeds a value of one because $\Delta \dot{n}_{\mathrm{I}}$ is calculated from the current assuming six electrons per molecule of $\mathrm{CO}_{2}$. The surface coverage is built up again during the negative half-wave causing a disproportionally large decrease in $\mathrm{CO}_{2}$ production rate.

The inductive behavior of $G_{\mathrm{I} / \mathrm{R}}$ at intermediate frequencies is also caused by surface processes, ${ }^{26,28}$ i.e. the interplay of the surface coverages of $\mathrm{CO}$ and $\mathrm{OH}$. When the potential rises, the $\mathrm{CO}$ coverage decreases. For the removal of one molecule of adsorbed CO, only two electrons are needed. Thus, the $\mathrm{CO}_{2}$ production rises faster than the current. When the potential falls again, the opposite occurs. Therefore, the maxima and minima of the reaction rate are located before the maxima and minima of the current. This corresponds to a negative phase shift and thus an inductive arc in the transfer function. The presence of the inductive arc shows that the model can structurally also reproduce an inductive loop in the EIS spectrum because the potential directly is coupled to the reaction rate when no capacitive effects are present.

The Nyquist plot for $G_{\mathrm{R} / \mathrm{M}}$, i.e. the transfer function relating $\mathrm{CO}_{2}$ transport through the membrane to reaction is depicted in Fig. 8b. The limiting magnitude at low frequency is different from the value one because only part of the $\mathrm{CO}_{2}$ that is produced diffuses to the membrane while the rest diffuses into the bulk electrolyte. Since the magnitudes of the other two transfer functions approach a limiting value of one at low frequencies, the magnitude of the overall sFRA transfer function at low frequencies is determined by this process. It does, however, not cause a large phase shift, as is evident from the small imaginary part. At high frequencies, $G_{\mathrm{R} / \mathrm{M}}$ converges again to zero, as the sinusoidal $\mathrm{CO}_{2}$ production rate is averaged out by the accumulation in the catalyst layer. The two arcs are produced by the interaction of the two transport processes into the membrane and into the bulk electrolyte with the accumulation of $\mathrm{CO}_{2}$ in the catalyst layer.

This can be confirmed by an estimation of the diffusion time constants $\tau$, which is given by the following equation

$$
\tau=\frac{\delta^{2}}{D^{\mathrm{eff}}}=\frac{\delta^{2}}{D \epsilon^{1.5}}
$$

with the characteristic diffusion length $\delta$, the $\mathrm{CO}_{2}$ diffusion coefficient $D$, the porosity $\epsilon$ and the effective diffusion coefficient $D^{\text {eff }}$. With the parameter values from Table A.I in the Appendix, the time constant of the catalyst layer $(0.37 \mathrm{~s})$, the membrane $(3.1 \mathrm{~s})$ and of the diffusion boundary layer $(5.2 \mathrm{~s})$ are calculated. The time constant of the catalyst layer corresponds to the characteristic frequency of $2 \mathrm{~Hz}$ of the first arc in the Nyquist plot and the time constants of boundary layer and membrane correspond to the characteristic frequency of the second arc of $0.074 \mathrm{~Hz}$. This analysis is confirmed by simulated spectra varying the membrane thickness $\delta^{\mathrm{M}}$, the thickness of the diffusion layer $\delta^{\mathrm{DL}}$ and the porosity of the catalyst layer $\epsilon^{\mathrm{AC}}$. In Fig. 8b it can be seen that a change in the first two parameters mainly affects the second arc, whereas a change in the latter parameter mainly affects the first arc.

In Fig. 8c, the Nyquist plot of the transfer function relating the MS signal to transport through the membrane is shown. $G_{\mathrm{M} / \mathrm{MS}}$ has a 


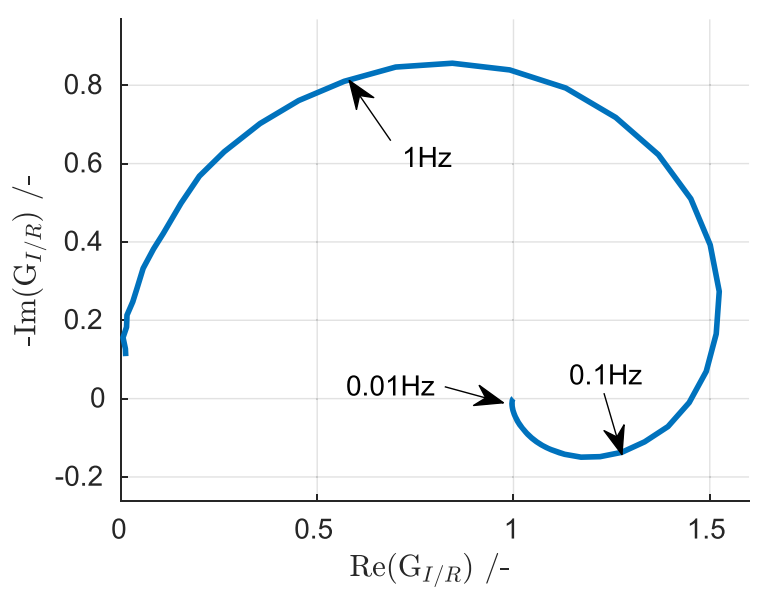

(a)

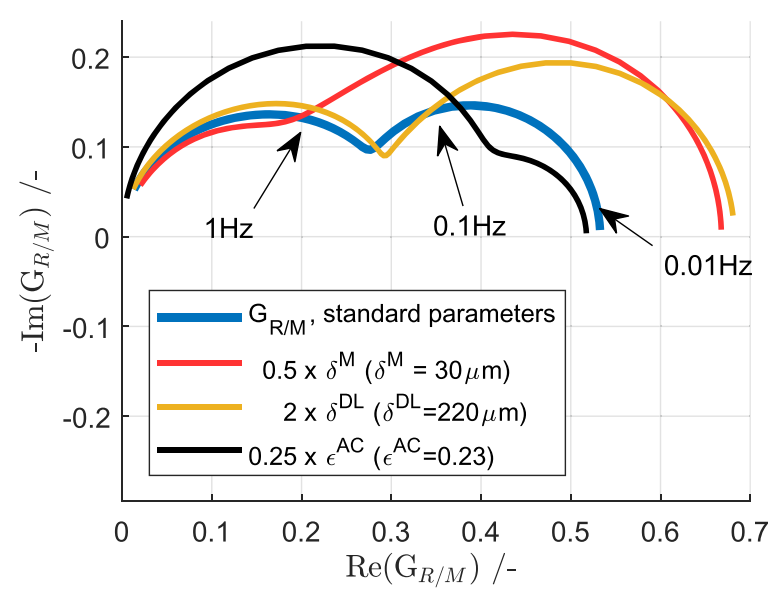

(b)

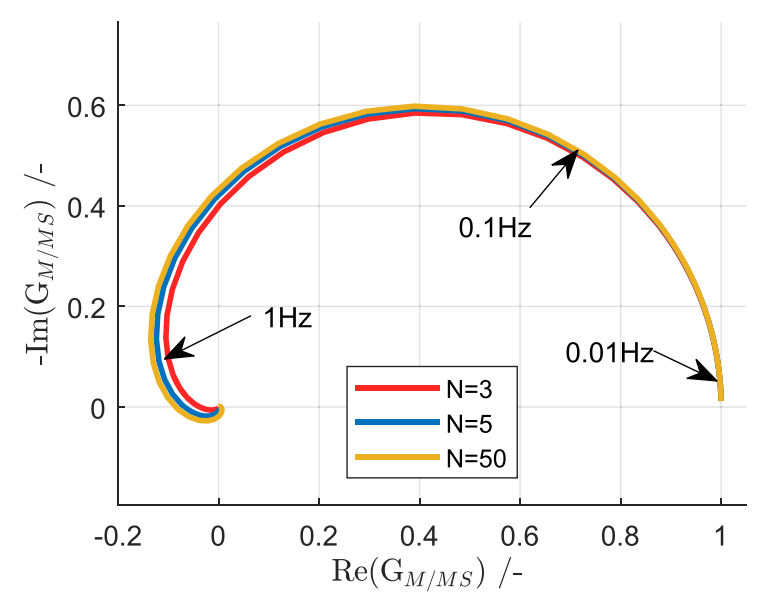

(c)

Figure 8. Simulated species FRA Nyquist plot of the transfer function relating: (a) the $\mathrm{CO}_{2}$ producing reaction to current $\left(G_{\mathrm{I} / \mathrm{R}}=\frac{\Delta \dot{n}_{\mathrm{COd}}^{\text {Prod }}}{\Delta \dot{n}_{I}}\right)$; (b) the transport from the reaction sites in the catalyst layer into the membrane $\left(G_{\mathrm{R} / \mathrm{M}}=\frac{\Delta \dot{n}_{\mathrm{M}}^{\text {in }}}{\Delta \dot{n}_{\mathrm{R}}}\right)$ with variation of transport properties; (c) the MS signal to transport through the membrane $\left(G_{\mathrm{M} / \mathrm{MS}}=\frac{\Delta \dot{n}_{\mathrm{MS}}}{\Delta \dot{n}_{\mathrm{M}}^{\text {in }}}\right)$ with different numbers of finite volume elements $N$ in the membrane. The frequency range is $0.002-10 \mathrm{~Hz}$.

limiting magnitude of one at low frequencies meaning that the flow of $\mathrm{CO}_{2}$ into the membrane equals the flow out of the membrane which is an important condition for obeying the law of mass conservation. The membrane transport causes negative real and imaginary values at higher frequencies. In the time domain (not shown) it can be seen that the phase shift increases continuously with increasing frequency. When the phase shift exceeds $\pi / 2$, a negative real part results. Such behavior may occur for partial differential equations that have more than one pole in the transfer function.

The physical process that causes the phase shift to increase continuously with the frequency is a dead time between the production and the detection of $\mathrm{CO}_{2}$. Regardless of the excitation signal's frequency, a certain amount of time is required for the diffusion of $\mathrm{CO}_{2}$ through the PTFE membrane. The phase shift $\Delta \phi$ equals the product of the (absolute) time difference between the two signals $\Delta t$ and the frequency $f: \Delta \phi=\Delta t \cdot f$. Thus, the phase shift approaches infinity for an infinitely large frequency.

The number of differential equations, i.e. discretisation elements of the membrane $N=5$, is comparatively low, considering the importance of the transport of $\mathrm{CO}_{2}$ through the membrane for the sFRA spectrum. Therefore, simulations with three different element numbers were carried out. The results are also shown in Fig. 8c. It can be seen that the discretisation of the membrane does not have an effect on the qualitative features of the curve. The chosen number of five elements is a compromise between simulation time and accuracy. As described in the Appendix, the parameter identification procedure limits the acceptable computational complexity of the model because thousands of spectra are simulated during the process.

The overall sFRA transfer function and the transfer functions of all processes discussed above are summarised in Fig. 9 to gain a better overview on the relative contributions of the sub-processes to the sFRA spectrum. The behavior at the lowest frequencies is exclusively determined by the transport from the catalyst into the membrane since the magnitude of the transfer functions of all other processes are one at these frequency values. At slightly higher frequencies, the inductive influence of the reaction and the capacitive influence of the membrane compensate each other. Although the magnitude of $G_{\mathrm{I} / \mathrm{R}}$ exceeds the value of one in an intermediate frequency range, this feature is not visible in the overall spectrum. The reason for this is that the transport and reaction processes both influence the intermediate frequency region. At the highest frequencies, the transport through the membrane dominates the spectrum.

In future work, the contributions of mass transfer to the transfer function might be assessed experimentally by using a reaction with very fast kinetics such as the hydrogen evolution reaction on platinum. Under these conditions, the transfer function $G_{\mathrm{I} / \mathrm{R}}$ will have a magnitude of one and no phase shift so that the overall transfer function is only influenced by mass transfer.

Sensitivity analysis.-Understanding the sensitivity of sFRA is important because only if sFRA is sensitive toward a parameter or process, information on this process can be deducted from the spectra. The goal of sFRA was the investigation of macrokinetics i.e. kinetics of electrode reactions influenced by transport. Thus, focus is placed on reaction and electrode transport parameters. To investigate which parameters affect the sFRA Nyquist curve, two relative sensitivity measures $S_{R}$ and $S_{f}$ are used as suggested by Grübl et al. ${ }^{12}$ :

$$
\begin{gathered}
S_{R}=\frac{R / R_{0}}{P / P_{0}} \\
S_{f}=\frac{f_{r} / f_{r, 0}}{P / P_{0}}
\end{gathered}
$$

$P_{0}$ is the nominal value of a parameter. $P$ is set $5 \%$ higher than $P_{0} . R$ is the difference in the real part of the transfer function between the lowest and the highest frequency for the parameter value $P$. Since the real part of the transfer function approaches zero 


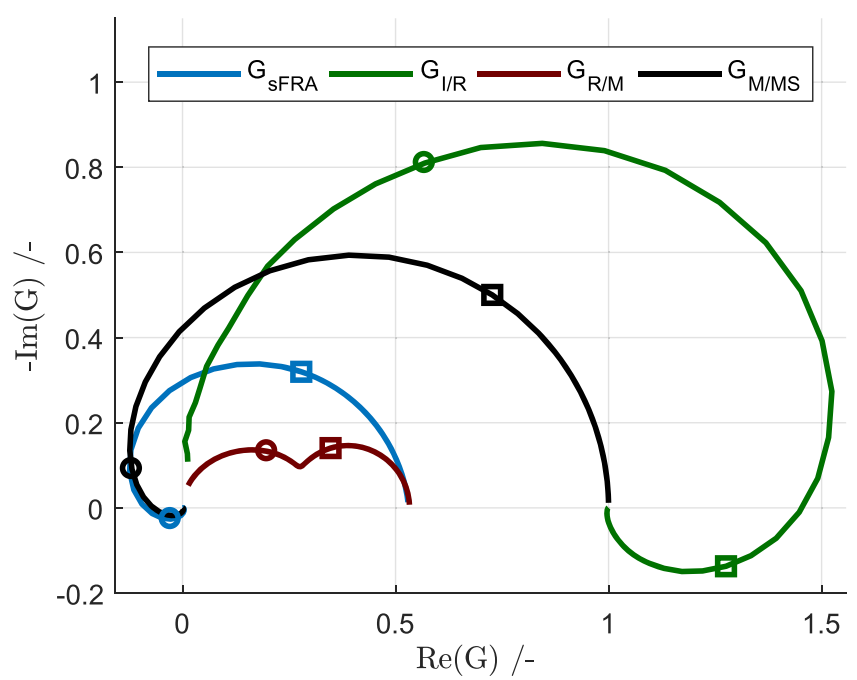

Figure 9. Decomposition of the sFRA Nyquist curve into the transfer functions of individual processes in a frequency range of $0.002-10 \mathrm{~Hz}$. The frequencies of $1 \mathrm{~Hz}$ and $0.1 \mathrm{~Hz}$ are marked with circles and squares, respectively.

at high frequencies and the imaginary part approaches zero at low frequencies, $R$ generally equals the magnitude of the transfer function at the lowest frequency. $f_{r}$ is the characteristic frequency where the imaginary part of the transfer function reaches its minimum for the parameter value $P$. Accordingly $f_{r, 0}$ and $R_{0}$ refer to the characteristic frequency and magnitude of the transfer function for the nominal parameter value $P_{0}$.

Thus $S_{R}$ describes the relative sensitivity of the magnitude at lowest frequency toward a parameter value $P$, whereas $S_{f}$ describes the relative sensitivity of the phase shift toward a parameter. While these two values do not capture the complete shape of the curves, they allow one to quantify and compare the effect of different parameters on the sFRA in terms of frequency and magnitude effects.

In Fig. 10, the relative sensitivities for selected geometric, transport and reaction parameters are shown.

There in no big difference in the sensitivity of the microkinetic parameters $C_{\mathrm{DL}}$ and $k_{3}$ between EIS and sFRA. The reason for this is that reaction rates and double layer capacitance affect the response of the potential and that of the species production rate in a similar manner. For instance, an increase in double layer capacitance buffers the effect of current density on potential as well as on reaction rate. Thus an increase in capacitance increases the time constant in sFRA and EIS which leads to a reduced characteristic frequency $f_{r}$ and a negative sensitivity $S_{\mathrm{f}}$ for $C_{\mathrm{DL}}$ in both sFRA and EIS. Thus, sFRA does not contain significantly more information on these parameters than EIS. For reactions with multiple gaseous reaction products, sFRA is expected to be far more favourable because spectra for the individual products can be recorded whereas the individual contributions of different reaction products to an EIS spectrum are hard to identify.

The picture is different for transport properties: Porosity and thickness of the catalyst layer ( $\epsilon^{\mathrm{AC}}$ and $\delta^{\mathrm{AC}}$ ) only have a negligible effect on EIS because the catalyst layer is thin enough to cause no mass transfer limitations for reactant supply. However, the catalyst layer properties have a strong effect on sFRA. They especially affect $f_{r}$. Larger thickness or larger pore volume fraction both increase the storage term inside the electrode causing a negative sensitivity of $S_{f}$ toward these values. Thus the method is indeed suitable to elucidate transport phenomena and to investigate macrokinetics of electrode reactions.

The characteristic frequency $f_{r}$ in sFRA is influenced by all parameters considered here. This finding agrees with the conclusions from the previous section because $f_{r}$ lies in an intermediate

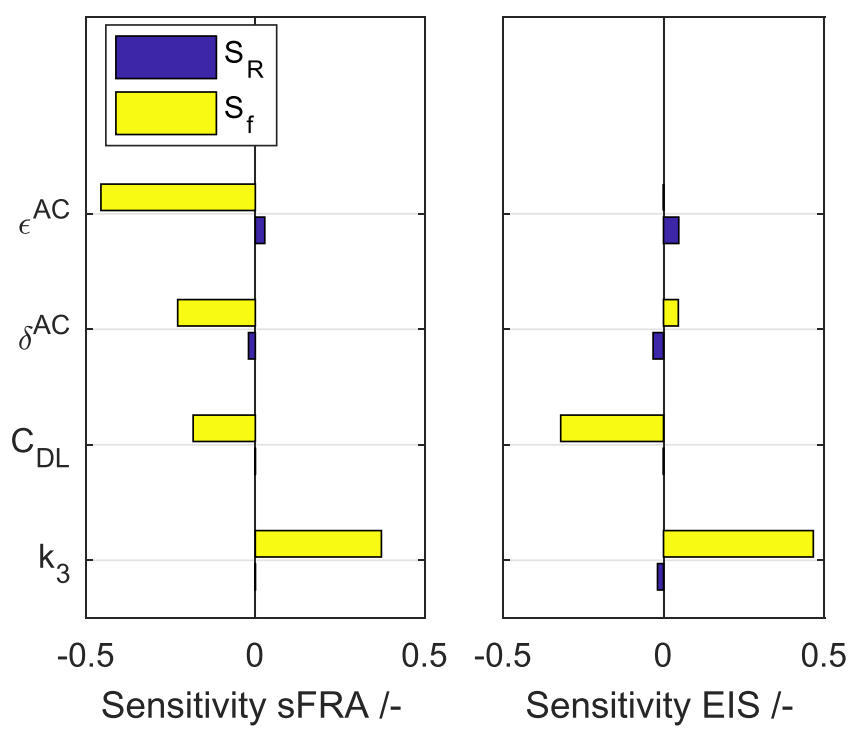

Figure 10. Sensitivities of sFRA Nyquist spectra on selected geometric, transport and reaction parameters.

frequency range near $0.1 \mathrm{~Hz}$ which is influenced by all processes. Because of this, the number of unknown parameters must not be too large in order to allow one to draw meaningful conclusions from the sFRA spectra.

\section{Conclusions}

The interpretation of sFRA spectra is still at the beginning, since this is the first time the methodology is applied. Still some general conclusions can be drawn from the analyses presented above:

- The frequency range of sFRA is limited at the upper end by the noise level of the MS signal and at the lower end by the time constants of the processes of interest.

- The maximum frequency can be increased when using larger current signal amplitudes because this increases the absolute amplitude of the MS signal. At high amplitudes, the benefits of increasing the amplitude further diminish.

- The simulations demonstrate that the current amplitude that is necessary for a frequency range up to $0.5 \mathrm{~Hz}$ causes small non-linear effects. With the help of the non-linear physical model it can be shown that the degree of non-linearity is small enough to approximate the system as linear. However, for analysing the spectra without a physical model, such an assessment of the linearity is not possible. Thus, the amplitude of the current signal cannot be increased arbitrarily and the characteristics of the electrochemical cell should be carefully considered when choosing the current signal amplitude. In this context potentiostatic operation is beneficial: it causes higher current amplitudes at high frequencies and thus increases the upper frequency range. At the same time, the current amplitudes are lower at lower frequencies where non-linear effects might occur.

- A sensitivity analysis confirms that sFRA is sensitive to transport parameters that are not accessible by EIS. It also showed that several processes overlap in an intermediate frequency range. Modelling can help to separate the contributions of individual processes to the sFRA spectrum.

Regarding the methanol oxidation reaction on a supported $\mathrm{Pt} / \mathrm{Ru}$ catalyst, which was used as an example to demonstrate the method, the reaction mechanism proposed in literature ${ }^{28}$ could be confirmed. Reaction rate constants for the individual reaction steps were determined at a temperature of $296 \mathrm{~K}$. Furthermore, it can be confirmed that the assumption of a homogeneous catalyst layer with effective diffusion coefficient following the Bruggeman 
equation is suitable to describe the transport within the electrode. Future work might elucidate if simpler kinetic models can be used to identify transport parameters in the electrode. This would be desirable to investigate new reactions where no mechanistic knowledge is available.

In its current state of development sFRA contains similar information on microkinetic parameters as EIS but additional information on transport parameters such as electrode porosity and effective diffusion coefficients in the electrode.

Perspectively, sFRA has one key advantage over other electrochemical techniques: if multiple electrochemical reactions take place, they can be differentiated. When using DEMS to measure the species flux, electrochemical reactions with more than one gaseous reactant such as the electrochemical $\mathrm{CO}_{2}$ reduction are promising future applications because the educt as well as volatile products such as $\mathrm{CO}$ and $\mathrm{H}_{2}$ may be distinguished.

\section{Acknowledgments}

The authors thank Dan Ye for programming the Labview software that was used for recording MSEIS data and Yuan Bo for assisting the model implementation.

\section{Appendix}

\section{A.1. Cyclone-flow DEMS cell}

A.2. Detailed model description.-In the following, the equations used to describe the reaction and transport processes during the MOR in the DEMS cell are explained.

The reaction mechanism and kinetics are modelled following a model from literature. ${ }^{28}$ It is described by Eqs. I-III in the main text. It has been shown that the changing surface coverages of adsorbed $\mathrm{CO}$ and $\mathrm{OH}$ have a significant influence on the MOR reaction kinetics. ${ }^{28}$ Thus the following balance equations for adsorbed $\mathrm{OH}$ and $\mathrm{CO}$ species are included, with $\theta_{\mathrm{CO} / \mathrm{OH}}$ denoting the relative surface coverage of the catalyst sites and $c_{\mathrm{Pt} / \mathrm{Ru}}$ denoting the number of available surface sites on the catalyst surface per unit of geometrical electrode surface area:

$$
\begin{aligned}
\frac{\mathrm{d} \theta_{\mathrm{CO}}}{\mathrm{d} t} & =\frac{1}{c_{\mathrm{Pt}}}\left(r_{1}-r_{3}\right) \\
\frac{\mathrm{d} \theta_{\mathrm{OH}}}{\mathrm{d} t} & =\frac{1}{c_{\mathrm{Ru}}}\left(r_{2}-r_{3}\right)
\end{aligned}
$$

The rates $r_{1}, r_{2}$ and $r_{3}$ of the three individual reaction steps (I), (II) and (III) are calculated with Frumkin/Temkin adsorption kinetics:

$$
\begin{gathered}
r_{1}=k_{10} \exp \left[-\beta_{\mathrm{CO}} g_{\mathrm{CO}}\left(\theta_{\mathrm{CO}}-0.5\right)\right] c_{\mathrm{CH}_{3} \mathrm{OH}}^{\mathrm{AC}}\left(1-\theta_{\mathrm{CO}}\right) \\
0 r_{2}=k_{20, f} \exp \left[\frac{\alpha \mathrm{F}}{\mathrm{R} T} \eta\right] \exp \left[-\beta_{\mathrm{OH}} g_{\mathrm{OH}}\left(\theta_{\mathrm{OH}}-0.5\right)\right]\left(1-\theta_{\mathrm{OH}}\right) \\
-k_{20, b} \exp \left[-\frac{(1-\alpha) \mathrm{F})}{\mathrm{R} T} \eta\right] \exp \left[\left(1-\beta_{\mathrm{OH}}\right) g_{\mathrm{OH}}\left(\theta_{\mathrm{OH}}-0.5\right)\right] \theta_{\mathrm{OH}} \\
r_{3}=k_{30} \exp \left[\left(1-\beta_{\mathrm{CO}}\right) g_{\mathrm{CO}}\left(\theta_{\mathrm{CO}}-0.5\right)\right] \theta_{\mathrm{CO}} \theta_{\mathrm{OH}}
\end{gathered}
$$

$g_{\mathrm{OH} / \mathrm{CO}}$ is the inhomogeneity/interaction factor for Frumkin/ Temkin adsorption for $\mathrm{OH}$ and $\mathrm{CO}, \alpha$ is the charge transfer coefficient, $k_{10}, k_{20, f}, k_{20, b}$, and $k_{30}$ are the reaction rate constants of the three reactions, and $\beta_{\mathrm{CO}}$ and $\beta_{\mathrm{OH}}$ is the symmetry factor for Frumkin/Temkin adsorption.

The reaction overpotential $\eta$ is calculated from a charge balance with the double layer capacity $C_{\mathrm{dl}}$ and the external cell current density $i_{\text {cell }}$ as in Eq. A.6. The sum of the overpotential and the reaction's equilibrium potential $E^{0}$ equals the electrode potential $E$ (Eq. A.7). The external potential $E_{\text {external }}$ that can be measured experimentally includes the uncompensated ohmic electrolyte resistance $R_{\mathrm{u}}$ :

$$
\begin{gathered}
\frac{\mathrm{d} \eta}{\mathrm{d} t}=\frac{1}{C_{\mathrm{dl}}} i_{\text {cell }}+\frac{1}{C_{\mathrm{dl}}}\left(-4 \mathrm{Fr} r_{1}-\mathrm{Fr}_{2}-F r_{3}\right) \\
E=\eta+E^{0} \\
E_{\text {external }}=E+R_{\mathrm{u}} i_{\text {cell }}
\end{gathered}
$$

The following balance equations describe the transport of methanol through the model domain depicted in Fig. 2:

$$
\begin{aligned}
& \frac{\mathrm{d} c_{\mathrm{CH}_{3} \mathrm{OH}}^{\mathrm{A}}}{\mathrm{d} t}=\frac{\dot{V}}{V^{\mathrm{A}}}\left(c_{\mathrm{CH}_{3} \mathrm{OH}}^{\mathrm{A} \text {,in }}-c_{\mathrm{CH}_{3} \mathrm{OH}}^{\mathrm{A}}\right) \\
& +\left.\frac{A_{\mathrm{el}}}{V^{\mathrm{A}}} D_{\mathrm{CH}_{3} \mathrm{OH}} \frac{\partial c_{\mathrm{CH}_{3} \mathrm{OH}}^{\mathrm{DL}}}{\partial x}\right|_{\mathrm{A} / \mathrm{DL}} \\
& \frac{\mathrm{d} c_{\mathrm{CH}_{3} \mathrm{OH}}^{\mathrm{DL}}}{\mathrm{d} t}=-D_{\mathrm{CH}_{3} \mathrm{OH}} \frac{\partial^{2} c_{\mathrm{CH}_{3} \mathrm{OH}}^{\mathrm{DL}}}{\partial x^{2}} \\
& \frac{\mathrm{d} c_{\mathrm{CH}_{3} \mathrm{OH}}^{\mathrm{AC}}}{\mathrm{d} t}=-D_{\mathrm{CH}_{3} \mathrm{OH}}^{\mathrm{AC}} \frac{\partial^{2} c_{\mathrm{CH}_{3} \mathrm{OH}}^{\mathrm{AD}}}{\partial x^{2}}-\frac{r_{1}}{\delta^{\mathrm{AC}}} \\
& \frac{\mathrm{d} c_{\mathrm{CH}_{3} \mathrm{OH}}^{\mathrm{M}}}{\mathrm{d} t}=-D_{\mathrm{CH}_{3} \mathrm{OH}}^{\mathrm{M}} \frac{\partial^{2} c_{\mathrm{CH}_{3} \mathrm{OH}}^{\mathrm{M}}}{\partial x^{2}}
\end{aligned}
$$

The superscripts A, DL, AC and $\mathrm{M}$ denote the respective domain as indicated in Fig. 2 with A/DL, DL/AC, and AC/M being the interfaces between anode bulk volume and diffusion layer, diffusion layer and anode catalyst layer, and anode catalyst layer and membrane. $\dot{V}$ is the volumetric flow rate, $c_{\mathrm{CH} 3 \mathrm{OH}}^{\mathrm{A}, \mathrm{i}}$ is the methanol feed concentration, $V^{\mathrm{A}}$ the anode chamber volume, $A^{\text {el }}$ is the electrode surface area. $D_{\mathrm{CH}_{3} \mathrm{OH}}^{\mathrm{M}}$ the diffusion coefficient of methanol through the membrane and $\mathrm{D}_{\mathrm{CH}}^{\mathrm{AC} O H}$ is the diffusion coefficient of methanol in the anode catalyst layer. $\delta^{\mathrm{AC}}$ is the thickness of the anode catalyst layer.

The thickness of the diffusion layer in front of the catalyst layer $\delta^{\mathrm{DL}}$ is obtained from a CFD analysis of the DEMS cell as described in our previous work. ${ }^{17} \mathrm{~A}$ detailed analysis and justification for treating the boundary layer as a layer of constant thickness with only diffusive transport can also be found there.

Transport of $\mathrm{CO}_{2}$ is crucial for the DEMS model. $\mathrm{CO}_{2}$ that is produced in the anode reaction can either diffuse back into the bulk electrolyte or through the PTFE membrane into the vacuum system. The following balance equations are established to describe the transport of $\mathrm{CO}_{2}$ :

$$
\begin{gathered}
\frac{\mathrm{d} c_{\mathrm{CO}_{2}}^{\mathrm{A}}}{\mathrm{d} t}=-\frac{\dot{V}}{V^{\mathrm{A}}} c_{C O_{2}}^{\mathrm{A}}+\left.\frac{A_{\mathrm{el}}}{V^{\mathrm{A}}} D_{C O_{2}} \frac{\partial c_{C O_{2}}^{\mathrm{DL}}}{\partial x}\right|_{\mathrm{A} / \mathrm{DL}} \\
\frac{\mathrm{d} c_{\mathrm{CO}_{2}}^{\mathrm{DL}}}{\mathrm{d} t}=-D_{\mathrm{CO}_{2}} \frac{\partial^{2} c_{C O_{2}}^{\mathrm{DL}}}{\partial x^{2}} \\
\frac{\mathrm{d} c_{C O_{2}}^{\mathrm{AC}}}{\mathrm{d} t}=-D_{C O_{2}}^{\mathrm{AC}} \frac{\partial^{2} c_{C O_{2}}^{\mathrm{DL}}}{\partial x^{2}}+\frac{r_{3}}{\delta^{\mathrm{AC}}} \\
\frac{\mathrm{d} c_{C O_{2}}^{\mathrm{M}}}{\mathrm{d} t}=-D_{\mathrm{CO}_{2}}^{\mathrm{M}} \frac{\partial^{2} c_{C O_{2}}^{\mathrm{M}}}{\partial x^{2}}
\end{gathered}
$$

Continuity of the $\mathrm{CO}_{2}$ and methanol flux is ensured at the boundaries between anode, anode diffusion layer, catalyst layer and membrane with the following boundary conditions: 


$$
\begin{aligned}
-\left.D_{\mathrm{CH}_{3} \mathrm{OH}} \frac{\partial c_{\mathrm{CH}_{3} \mathrm{OH}}^{\mathrm{DL}}}{\partial x}\right|_{\mathrm{DL} / \mathrm{AC}} & =-\left.D_{\mathrm{CH}_{3} \mathrm{OH}}^{\mathrm{AC}} \frac{\partial c_{\mathrm{CH}_{3} \mathrm{OH}}^{\mathrm{AC}}}{\partial x}\right|_{\mathrm{DL} / \mathrm{AC}} \\
-\left.D_{\mathrm{CO}_{2}} \frac{\partial c_{\mathrm{CO}_{2}}^{\mathrm{DL}}}{\partial x}\right|_{\mathrm{DL} / \mathrm{AC}} & =-\left.D_{\mathrm{CO}_{2}}^{\mathrm{AC}} \frac{\partial c_{\mathrm{CO}_{2}}^{\mathrm{AC}}}{\partial x}\right|_{\mathrm{DL} / \mathrm{AC}} \\
-\left.D_{\mathrm{CH}_{3} O H}^{\mathrm{AC}} \frac{\partial c_{\mathrm{CH}_{3} \mathrm{OH}}^{\mathrm{AC}}}{\partial x}\right|_{\mathrm{AC} / \mathrm{M}} & =-\left.D_{\mathrm{CH}_{3} O H}^{\mathrm{M}} \frac{\partial c_{\mathrm{CH}_{3} O H}^{\mathrm{O}}}{\partial x}\right|_{\mathrm{AC} / \mathrm{M}} \\
-\left.D_{\mathrm{CO}}^{\mathrm{AC}} \frac{\partial c_{\mathrm{CO}}^{\mathrm{AC}}}{\partial x}\right|_{\mathrm{AC} / \mathrm{M}} & =-\left.D_{\mathrm{CO}_{2}}^{\mathrm{M}} \frac{\partial c_{\mathrm{CO}}^{\mathrm{M}}}{\partial x}\right|_{\mathrm{AC} / \mathrm{M}}
\end{aligned}
$$

At the membrane/vacuum interface (see Fig. 2), the $\mathrm{CO}_{2}$ and methanol concentrations are set to zero because the partial pressures in the vacuum can be neglected.

The flux of $\mathrm{CO}_{2}$ into the vacuum $\dot{n}_{\mathrm{MS}}$ is calculated from the concentration gradient at the interface $\mathrm{M} / \mathrm{Vac}$ between membrane and vacuum:

$$
\dot{n}_{\mathrm{MS}}=-\left.A D_{\mathrm{CO}_{2}} \frac{\partial c_{\mathrm{CO}_{2}}^{\mathrm{M}}}{\partial x}\right|_{\mathrm{M} / \mathrm{Vac}}
$$

Diffusion coefficients of $\mathrm{CO}_{2}$ and $\mathrm{CH}_{3} \mathrm{OH}$ in the porous catalyst layer are calculated via the Bruggemann equation:

$$
D_{j}^{\mathrm{AC}}=D_{j}\left(\varepsilon^{\mathrm{AC}}\right)^{1.5}
$$

Diffusion coefficients of methanol and $\mathrm{CO}_{2}$ in the membrane are identified from experimental data. This approach is chosen because the representation of transport through the membrane as diffusive transport is a simplification of the physical process. At the vacuum side of the membrane, where the pressure in the pores is very low, transport might not be diffusive any more. Exploring the membrane transport in detail is, however, beyond the scope of this work.

The values of all fixed model parameters are reported in Table A.I. The equations were implemented in Matlab. Transport equations were solved with a finite volume scheme using $N_{\text {elements }}^{\mathrm{DL}}=5$ volume elements for the diffusion layer, $N_{\text {elements }}^{\mathrm{AC}}=5$ volume elements for the catalyst layer, and $N_{\text {elements }}^{\mathrm{M}}=5$ volume elements for the membrane. This comparatively low number of elements is chosen to limit the computational cost of the parameter identification that will be explained in the next section. The impact of the number of volume elements on the simulation results is discussed in the results section. Among the simulation outputs are the responses to a sinusoidal excitation signal in the time domain. The magnitude and phase shift of the response signals are obtained by fitting a sine function to the response signals. From the amplitude and phase shift of the sine function, the Nyquist plots are constructed.

A.3. Parameter identification.-The parameters for the MOR model from Krewer et al. ${ }^{28}$ were determined for a temperature of $343 \mathrm{~K}$ whereas experiments in this work were carried out at a temperature of $296 \mathrm{~K}$. Furthermore the morphology of the used catalyst particles may be different. Therefore, kinetic rate constants from Krewer et al. cannot be used. Also, as explained above, diffusion coefficients in the porous PTFE membrane are treated as additional fitting parameters.

The model parameters $\theta$ are identified by minimizing the deviation between experimental and simulation results using the following objective function $f$ :
Table A·I. Fixed model parameters.

\begin{tabular}{lcl} 
Parameter & Value & Notes \\
\hline$\delta^{\mathrm{DL}} / \mathrm{m}$ & $110 \cdot 10^{-6}$ & Ref. 17 \\
$\delta^{\mathrm{M} / \mathrm{m}}$ & $60 \cdot 10^{-6}$ & Ref. 17 \\
$\delta^{\mathrm{AC}} / \mathrm{m}$ & $25 \cdot 10^{-6}$ & Ref. 17 \\
$\epsilon^{\mathrm{AC}} /-$ & 0.92 & Ref. 17 \\
$\epsilon^{\mathrm{M}} /-$ & 0.72 & Ref. 17 \\
$\dot{V} / 1 \mathrm{~s}^{-1}$ & $3.67 \cdot 10^{-3}$ & experiment \\
$A_{\mathrm{el}} / \mathrm{m}^{2}$ & $78.5 \cdot 10^{-6}$ & experiment \\
$c_{\mathrm{CH} 3 \mathrm{OH}}^{\text {in }} / \mathrm{mol} \mathrm{m}^{-3}$ & 500 & experiment \\
$T / \mathrm{K}$ & 298 & experiment \\
$E^{0} / \mathrm{V}$ & -0.036 & Nernst equation \\
& & with standard \\
& & potential from Ref. 25 \\
$D_{\mathrm{CH} 3 \mathrm{OH}} / \mathrm{m}^{2} \mathrm{~s}^{-1}$ & $0.84 \cdot 10^{-9}$ & Ref. 32 \\
$D_{\mathrm{CO} 2} / \mathrm{m}^{2} \mathrm{~s}^{-1}$ & $1.92 \cdot 10^{-9}$ & Ref. 32 \\
$g_{\mathrm{OH}} /-$ & 0.43 & Ref. 28 \\
$g_{\mathrm{CO}} /-$ & 11 & Ref. 28 \\
$\beta_{\mathrm{OH}} /-$ & 0.5 & Ref. 28 \\
$\beta_{\mathrm{CO} /} /-$ & 0.5 & Ref. 28 \\
$\alpha /-$ & 0.5 & Ref. 28 \\
$N_{\text {elements }}^{\mathrm{DL}} /-$ & 5 & \\
$N_{\text {elements }}^{\mathrm{AC}} /-$ & 5 & \\
$N_{\text {elements }}^{\mathrm{M}} /-$ & 5 &
\end{tabular}

$$
\begin{gathered}
\min _{\theta} f=\epsilon_{\mathrm{CV}}+\epsilon_{\mathrm{FRA}} \\
\epsilon_{\mathrm{CV}}=\frac{W_{\mathrm{CV}, \mathrm{i}}}{n_{\mathrm{CV}}} \sum_{i=1}^{n_{\mathrm{CV}}}\left(I_{i}^{\exp }-I_{i}^{\mathrm{sim}}\right)^{2} \\
+\frac{W_{\mathrm{CV}, \mathrm{MS}}}{n_{\mathrm{CV}}} \sum_{j=1}^{n_{\mathrm{CV}}}\left(I_{\mathrm{MS}, j}^{\exp }-I_{\mathrm{MS}, j}^{\operatorname{sim}}\right)^{2} \\
\epsilon_{\mathrm{FRA}}=\frac{W_{\mathrm{FRA}, \mathrm{MS}}}{n_{\mathrm{FRA}}} \sum_{i=1}^{n_{\mathrm{FRA}}}\left(\operatorname{Im}\left(G_{\mathrm{MS}, i}^{\exp }\right)-\operatorname{Im}\left(G_{\mathrm{MS}, i}^{\operatorname{sim}}\right)\right)^{2}+ \\
\left(\operatorname{Re}\left(G_{\mathrm{MS}, i}^{\exp }\right)-\operatorname{Re}\left(G_{\mathrm{MS}, i}^{\operatorname{sim}}\right)\right)^{2}+\frac{W_{\mathrm{FRA}, \mathrm{i}} \sum_{\mathrm{FRA}}\left(\operatorname{Im}\left(Z_{i}^{\exp }\right)-\operatorname{Im}\left(Z_{i}^{\operatorname{sim}}\right)\right)^{2}+}{n_{\mathrm{FRA}}}{ }_{i=1}^{[\mathrm{A} \cdot 26} \\
\left(\operatorname{Re}\left(Z_{i}^{\exp }\right)-\operatorname{Re}\left(Z_{i}^{\operatorname{sim}}\right)\right)^{2}
\end{gathered}
$$

With $\epsilon_{\mathrm{FRA}}, \epsilon_{\mathrm{CV}}$ denoting the deviation between simulated and experimental Nyquist and CV curves. $\epsilon_{\mathrm{FRA}}$ and $\epsilon_{\mathrm{CV}}$ are calculated by summing up the squared differences between experimental and simulated mass spectrometric and electrochemical data.

For the $\mathrm{CV}$, the squared deviations between experiment and simulation of current $i_{i}$ and ion current $I_{\mathrm{MS}, \mathrm{i}}$ are directly summed up over the $n_{\mathrm{CV}}$ data points that were recorded. The weight factors $W_{\mathrm{CV}, \mathrm{i}}, W_{\mathrm{CV}, \mathrm{MS}}, W_{\mathrm{FRA}, \mathrm{i}}$ and $W_{\mathrm{FRA}, \mathrm{MS}}$ take into account that the ion current and current signals differ by several orders of magnitude ( $\mathrm{mA}$ vs nA) and allow to place a different weight on the two experiments.

For the sFRA and EIS spectra, the deviation between experiment and simulation is calculated from the squared distances between the experimental and the simulated Nyquist curve that are summed up over the number of frequencies $n_{\mathrm{FRA}}$.

The objective function is minimised by the genetic algorithm from Matlab's global optimization toolbox because gradient-based 


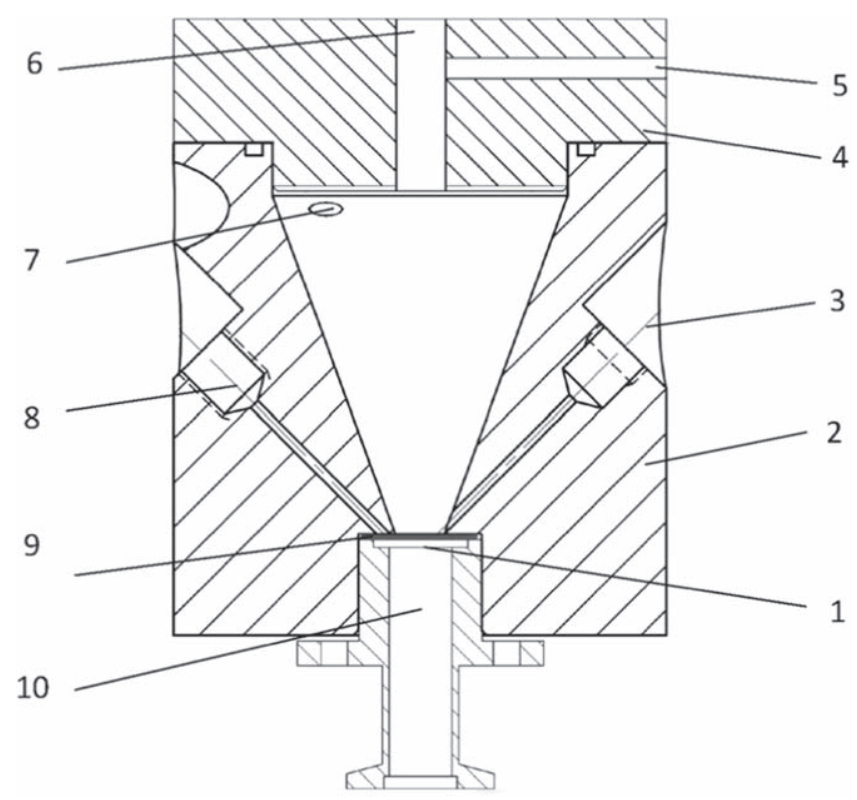

Figure A $\mathbf{1}$. Design of the cyclone flow DEMS cell, including: (1) stainless steel frit, (2) cell body from Kel-F, (3) connection to reference electrode, (4) cell cap from Kel-F, (5) electrolyte outlet, (6) connection to counter electrode, (7) tangential electrolyte inlet, (8) Pt-wire as current collector, (9) working electrode on porous PTFE membrane and gasket, (10) connection to vacuum system/MS. Reprinted from Ref. 17 with permission from Elsevier.

algorithms were only able to find local optima of the objective function. The following values were used for the weight factors: $W_{\mathrm{CV}, \mathrm{i}}=4.86 \cdot 10^{5} \mathrm{~A}^{-2}, W_{\mathrm{CV}, \mathrm{MS}}=5.6 \cdot 10^{19} \mathrm{~A}^{-2}, W_{\mathrm{FRA}, \mathrm{i}}=0.12 \Omega^{-2}$ and $W_{\text {FRA,MS }}=2.68 \cdot 10^{15}$. The weights were obtained by trial and error and the quality of the fit was evaluated visually.

The genetic algorithm typically needs about 80.000 evaluations of the objective function before converging which is reasonable given the number of parameters. Since every evaluation of the objective function includes the simulation of a mass spectrometric cyclic voltammogram (MSCV) and a simulation of the response to a sinusoidal input signal for each frequency, a computer with $8 \mathrm{~GB}$ of RAM and an i7 Quad-Core CPU needs about $15 \mathrm{~h}$ for the parameter identification even though a single simulation only takes one to two seconds.

A.4. Model validation.-A single parameter set was identified for the CV and FRA experiments using the procedure described above. As discussed in the main body of the text, experimental and simulated EIS and sFRA spectra match well for the identified parameter set.

In Fig. A.2, the experimental and the simulated CV and MSCV are compared. The overall shape of the curves matches well. Experimental current density, $\mathrm{CO}_{2}$ flux, which is calculated from the ion current signal, and the MS calibration constant, are well reproduced by the model with the identified parameter set. Because of the low scan rate the CV does not show many characteristic features for CVs. The main deviation between experiment and simulation results from the fact that the experimental values of current density and $\mathrm{CO}_{2}$ flux do not return to their starting value at the end of the cycle. The reason for this might be a shift of the ion current background.

All together, the simulation model reproduces CV, MSCV, EIS, and SFRA spectra comparatively well with a single parameter set.

In Fig. A.3, the simulated surface coverages of $\mathrm{CO}$ and $\mathrm{OH}$ during the $\mathrm{CV}$ are shown. The coverage of $\mathrm{CO}$ continuously decreases with potential. The $\mathrm{OH}$ coverage only increases at potentials above $0.65 \mathrm{~V}$. The coverage of $\mathrm{CO}$ exhibits some hysteresis because in the positive scan, when the removal rate of
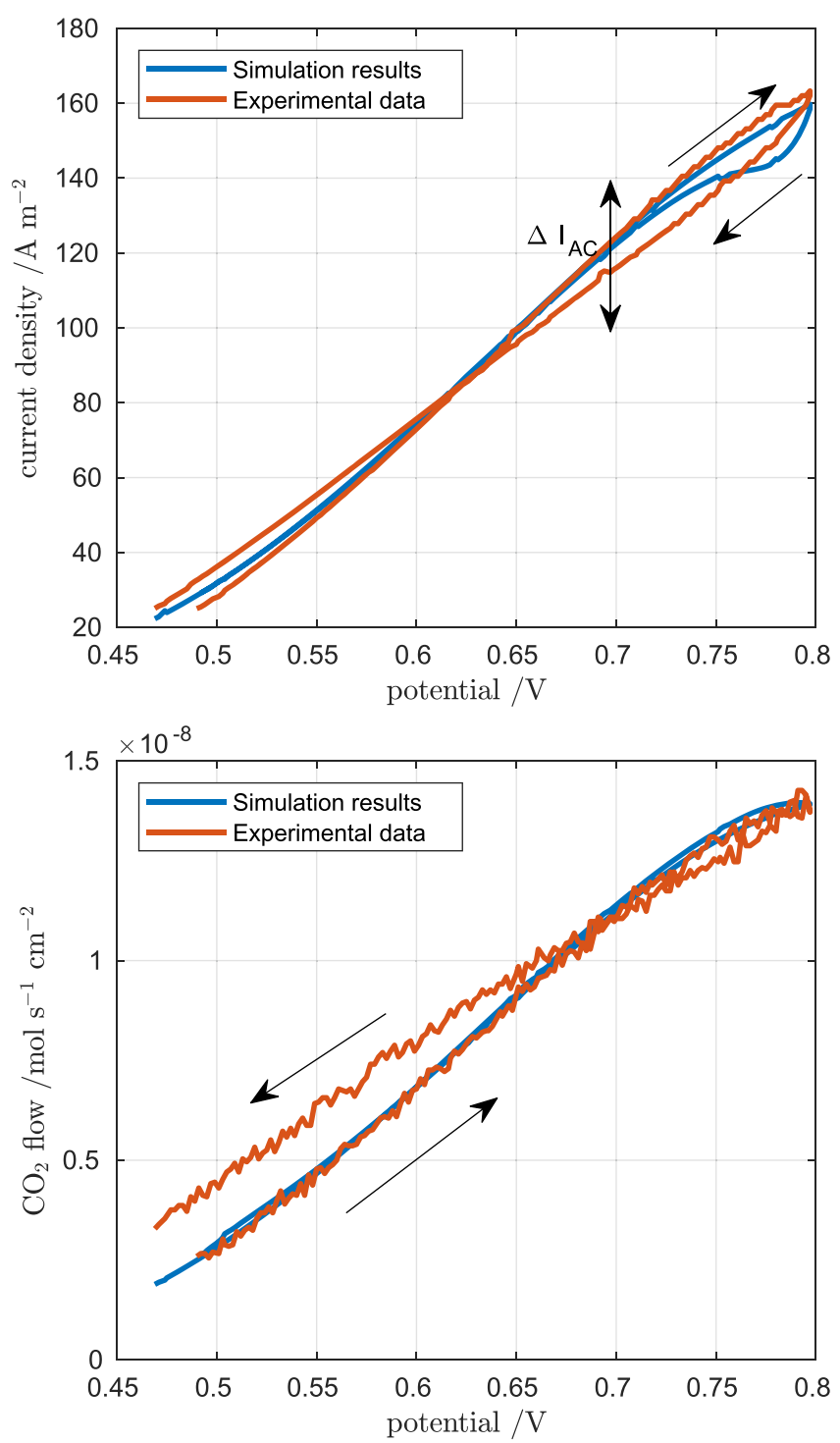

Figure A-2. Comparison of experiment and simulation for $\mathrm{CV}$ and $\mathrm{MSCV}$. Scan rate $2 \mathrm{mV} \mathrm{s}^{-1}, c_{\mathrm{MeOH}}=0.5 \mathrm{~mol}^{-1}$ in $0.1 \mathrm{~mol}^{-1} \mathrm{HClO}_{4}, 0.5 \mathrm{mg} \mathrm{cm}^{-2}$ $\mathrm{Pt} / \mathrm{Ru}$ on carbon, $\mathrm{T}=25^{\circ} \mathrm{C}$.

$\mathrm{CO}$ from the surface is increasing, some time is needed before the surface coverage follows the potential. In the negative scan a delay occurs because the dissociative adsorption of methanol, that increases $\mathrm{CO}$ coverage, takes some time. The overall trend agrees to results found in the literature. The hysteresis is relatively small because of the low scan rate of $2 \mathrm{mV} \mathrm{s}^{-1}$.

In Table A.II, the values of the identified model parameters and the parameter values from Krewer et al. ${ }^{28}$ are shown. It can be seen that the identified kinetic parameter values differ strongly from the values found there. The reasons for the deviation might be the difference between half cell and full cell set up, the different amounts of catalyst and its preparation, the difference between liquid and solid electrolyte, or the different temperature. The double layer capacitance and number of surface sites show large differences when normalised by the geometric surface area of the electrode. However, when accounting for the fact that the catalyst loading in Ref. 28 was 16 times larger than in this study, the numbers of surface sites per gram of catalyst are on the same order of magnitude and the double layer capacitance agrees with literature values. The value of the diffusion coefficient of $\mathrm{CO}_{2}$ in the membrane is very close to the value calculated via the Bruggeman equation from the membrane porosity and the diffusion coefficient of $\mathrm{CO}_{2}$ in the 


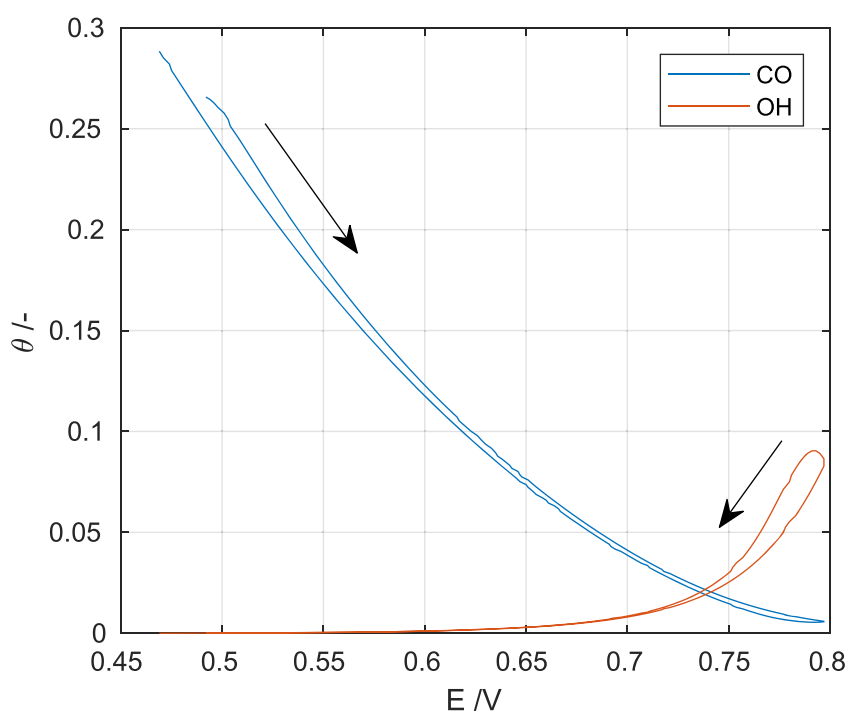

Figure A.3. Simulated surface coverages of $\mathrm{CO}$ and $\mathrm{OH}$ as a function of the potential for $\mathrm{CV}$ and $\mathrm{MSCV}$, scan rate $2 \mathrm{mV} \mathrm{s}^{-1}, c_{\mathrm{MeOH}}=0.5 \mathrm{~mol}^{-1}$ in $0.1 \mathrm{~mol}^{-1} \mathrm{HClO}_{4}, 0.5 \mathrm{mg} \mathrm{cm}^{-2} \mathrm{Pt} / \mathrm{Ru}$ on carbon, $\mathrm{T}=25^{\circ} \mathrm{C}$.

\section{Table A.II. Comparison of identified parameter values for MOR on} $\mathrm{Pt} / \mathrm{Ru}$ and literature values.

$\begin{array}{lcc}\text { Parameter } & \text { Best fit } & \text { Krewer et al. }{ }^{28} \\ k_{10} / \mathrm{m} \mathrm{s}^{-1} & 8.12 \cdot 10^{-8} & 1.6 \cdot 10^{-4} \\ k_{20 \mathrm{f}} / \mathrm{mol} \mathrm{m}^{-2} \mathrm{~s}^{-1} & 1.43 \cdot 10^{-6} & 7.2 \cdot 10^{-4} \\ r_{20 \mathrm{~b}} / \mathrm{mol} \mathrm{m}^{-2} \mathrm{~s}^{-1} & 1.59 \cdot 10^{7} & 9.91 \cdot 10^{-4} \\ r_{30} / \mathrm{mol} \mathrm{m}^{-2} \mathrm{~s}^{-1} & 8.07 & 0.19 \\ C_{\mathrm{dl}} / \mathrm{F} \mathrm{m}^{-2} & 226.6 & 3348 \\ \left(\mathrm{~F} \mathrm{~g}_{\text {catalyst }}^{-1}\right) & (55.3) & (41.9) \\ R_{\mathrm{ohm}} / \Omega & 12.3 & - \\ c_{\mathrm{P}} / \mathrm{mol} \mathrm{m}^{-2} & 2.65 \cdot 10^{-3} & 0.117 \\ \left(\mathrm{~mol} \mathrm{~g} \text { catalyst }^{-1}\right) & \left(0.53 \cdot 10^{-3}\right) & \left(2.06 \cdot 10^{-3}\right) \\ c_{\mathrm{Ru}} / \mathrm{mol} \mathrm{m}^{-2} & 0.018 & 0.165 \\ \left(\mathrm{~mol} \mathrm{~g} \mathrm{c}_{\text {catalyst }}^{-1}\right) & \left(3.6 \cdot 10^{-3}\right) & \left(1.46 \cdot 10^{-3}\right) \\ D_{\mathrm{CH} 3 \mathrm{OH}}^{\mathrm{M}} / \mathrm{m}^{2} \mathrm{~s}^{-1} & 5.70 \cdot 10^{-10} & 6.26 \cdot 10^{-10 \mathrm{a})} \\ D_{\mathrm{CO} 2}^{\mathrm{M}} / \mathrm{m}^{2} \mathrm{~s}^{-1} & 1.26 \cdot 10^{-9} & - \\ \mathrm{a})=\mathrm{in} \mathrm{Nafion} & & \end{array}$

electrolyte of $1.17 \cdot 10^{-9} \mathrm{~m}^{2} \mathrm{~s}^{-1} \cdot{ }^{17}$ Also, the value of the diffusion coefficient of methanol in the membrane agrees well with the value calculated via the Bruggeman equation from the diffusion coefficient of methanol in the electrolyte of $5.13 \cdot 10^{-10} \mathrm{~m}^{2} \mathrm{~s}^{-1}$. Thus the identification of the diffusion coefficients might not be necessary and literature values could be used in future studies.

\section{ORCID}

Fabian Kubannek (D) https://orcid.org/0000-0002-9513-0179 Ulrike Krewer (iD https://orcid.org/0000-0002-5984-5935

\section{References}

1. F. Kubannek, T. Turek, and U. Krewer, Chemie Ingenieur Technik, 91, 720 (2019).

2. N. Wolff, N. Harting, F. Röder, M. Heinrich, and U. Krewer, The European Physical Journal Special Topics, 227, 2617 (2019).

3. B. Bensmann, M. Petkovska, T. Vidakovic-Koch, R. Hanke-Rauschenbach, and K. Sundmacher, J. Electrochem. Soc., 157, B1279 (2010).

4. Q. Mao, U. Krewer, and R. Hanke-Rauschenbach, Electrochemistry Communications, 12, 1517 (2010)

5. Q. Mao and U. Krewer, Electrochimica Acta, 103, 188 (2013)

6. T. Kadyk, R. Hanke-Rauschenbach, and K. Sundmacher, Journal of Applied Electrochemistry, 41, 1021 (2011).

7. N. Wolff, N. Harting, M. Heinrich, and U. Krewer, Electrochimica Acta, 298, 788 (2018).

8. I. Citti, O. Aaboubi, J. P. Chopart, C. Gabrielli, A. Olivier, and B. Tribollet, J. Electrochem. Soc., 144, 2263 (2019).

9. Z. Rotenberg, Electrochimica Acta, 42, 793 (1997)

10. L. M. Peter, J. Li, R. Peat, H. Lewerenz, and J. Stumper, Electrochimica Acta, 35, 1657 (1990).

11. P. Hartmann, D. Grübl, H. Sommer, J. Janek, W. G. Bessler, and P. Adelhelm, The Journal of Physical Chemistry C, 118, 1461 (2014).

12. D. Grübl, J. Janek, and W. G. Bessler, J. Electrochem. Soc., 163, A599 (2016).

13. E. Engebretsen, T. J. Mason, P. R. Shearing, G. Hinds, and D. J. Brett Electrochemistry Communications, 75, 60 (2017).

14. A. Kulikovsky, eTransportation, 2, 100026 (2019).

15. A. Sorrentino, T. Vidakovic-Koch, and K. Sundmacher, Journal of Power Sources, 412, 331 (2019).

16. M. Röhe, F. Kubannek, and U. Krewer, Chem. Sus. Chem, 12, 2373 (2019).

17. F. Kubannek and U. Krewer, Electrochimica Acta, 210, 862 (2016).

18. H. Wang, C. Wingender, H. Baltruschat, M. Lopez, and M. Reetz, Journal of Electroanalytical Chemistry, 509, 163 (2001).

19. H. Wang, L. R. Alden, F. J. DiSalvo, and H. D. Abruña, Langmuir, 25, 7725 (2009)

20. H. Wang, E. Rus, and H. D. Abruña, Anal. Chem., 82, 4319 (2010).

21. S. Wasmus, J.-T. Wang, and R. F. Savinell, J. Electrochem. Soc., 142, 3825 (1995).

22. T. Seiler, E. Savinova, K. A. Friedrich, and U. Stimming, Electrochimica Acta, 49 , 3927 (2004).

23. S. Sakong and A. Groß, Electrocatalysis, 8, 577 (2017).

24. M. Metikoš-Hukovic, R. Babic, and Y. Piljac, Journal of New Materials for Electrochemical Systems, 7, 179 (2004).

25. T. Iwasita, Electrochimica Acta, 47, 3663 (2002).

26. U. Krewer, M. Christov, T. Vidaković, and K. Sundmacher, Journal of Electroanalytical Chemistry, 589, 148 (2006).

27. U. Krewer, A. Kamat, and K. Sundmacher, Journal of Electroanalytical Chemistry, 609, 105 (2007).

28. U. Krewer, H.-K. Yoon, and H.-T. Kim, Journal of Power Sources, 175, 760 (2008).

29. T. Vidaković, M. Christov, and K. Sundmacher, Journal of Electroanalytical Chemistry, 580, 105 (2005).

30. N. Wolff, N. Harting, M. Heinrich, F. Röder, and U. Krewer, Electrochimica Acta, 260, 614 (2018).

31. U. Krewer and K. Sundmacher, Journal of Power Sources, 154, 153 (2006).

32. E. L. Cussler, Diffusion: Mass Transfer in Fluid Systems (Cambridge University Press, New York) 2nd ed. (1997). 